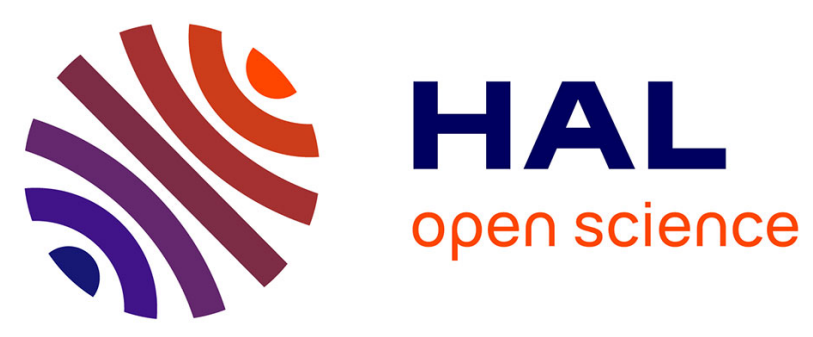

\title{
Effective model for elastic waves in a substrate supporting a array of plates/beams with flexural and longitudinal resonances
}

Jean-Jacques Marigo, Kim Pham, Agnès Maurel, Sébastien Guenneau

\section{To cite this version:}

Jean-Jacques Marigo, Kim Pham, Agnès Maurel, Sébastien Guenneau. Effective model for elastic waves in a substrate supporting a array of plates/beams with flexural and longitudinal resonances. Journal of Elasticity, 2021, 146 (1), pp.143-177. 10.1007/s10659-021-09854-4 . hal-03431828

\author{
HAL Id: hal-03431828 \\ https://hal.science/hal-03431828
}

Submitted on 16 Nov 2021

HAL is a multi-disciplinary open access archive for the deposit and dissemination of scientific research documents, whether they are published or not. The documents may come from teaching and research institutions in France or abroad, or from public or private research centers.
L'archive ouverte pluridisciplinaire $\mathbf{H A L}$, est destinée au dépôt et à la diffusion de documents scientifiques de niveau recherche, publiés ou non, émanant des établissements d'enseignement et de recherche français ou étrangers, des laboratoires publics ou privés. 


\title{
Effective model for elastic waves in a substrate supporting a array of plates/beams with flexural and longitudinal resonances
}

\author{
Jean-Jacques Marigo \\ Laboratoire de Mécanique des solides, Ecole Polytechnique, \\ Route de Saclay, 91120 Palaiseau, France \\ Kim Pham \\ IMSIA, ENSTA ParisTech - CNRS - EDF - CEA, Université Paris-Saclay, \\ 828 Bd des Maréchaux, 91732 Palaiseau, France \\ Agnès Maurel \\ Institut Langevin, ESPCI Paris, Université PSL, CNRS, \\ 1 rue Jussieu, Paris 75005, France \\ Sébastien Guenneau \\ Aix Marseille Univ, CNRS, Centrale Marseille, Institut Fresnel, Marseille, France
}

\begin{abstract}
In a previous study [1] we have studied the effect of an array of plates or beams over an elastic ground on the propagation of waves hitting the interface. The study was restricted to the low frequency regime where only flexural resonances take place. The present study presents a generalization to higher frequencies which allows us to account for both flexural and longitudinal resonances and to evaluate their interplay. An effective model is obtained using asymptotic analysis and homogenization techniques, which can be expressed in terms of the ground alone with an effective dynamic (frequency-dependent) boundary conditions of the Robin's type. For an inplane wave at oblique incidence, the scattered displacement fields and the reflection coefficients are obtained in closed forms and their effectiveness to reproduce the actual scattering is inspected by comparison with direct numerics in a two-dimensional setting.
\end{abstract}

Keywords: asymptotic analysis; elastic waves; metamaterials; metasurfaces; multimodal methods.

2010 MSC: 00-01, 99-00

\section{Introduction}

The ability of arrays of resonant beams to modify the propagation of waves in a substrate which support them is now well established. Their study, primarily focused on the case of a plate substrate, have been then extended to the case of a half-plane substrate. In both cases, massspring oscillator models have been used as the simplest models, see e.g. [2, 3, 4]. Assuming point contacts between the resonators and the substrate, the displacement $X$ of a single oscillator 


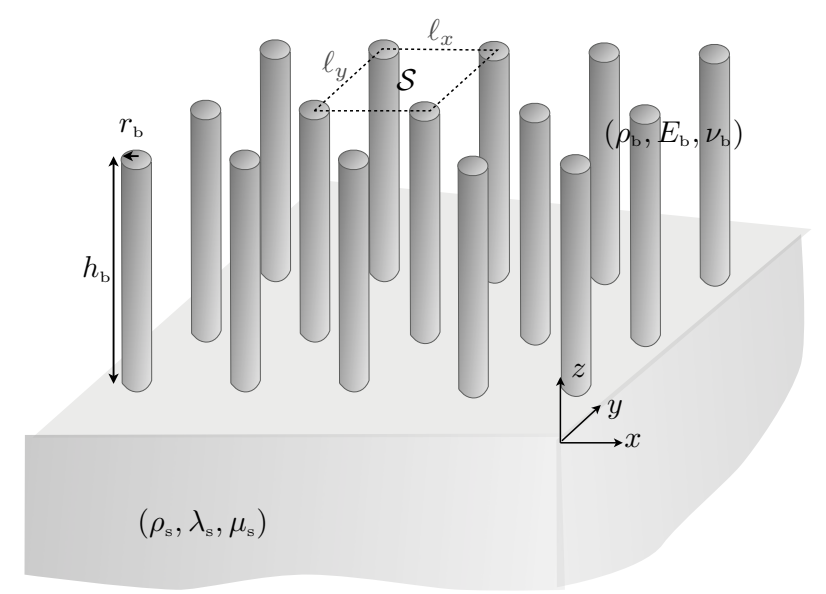

Figure 1: Array of cylindrical beams atop an isotropic substrate submitted to bending and longitudinal resonances; with $k_{\mathrm{T}}$ the wavenumber in the substrate, $k_{\mathrm{T}} h_{\mathrm{b}}=O(1)$ and $k_{\mathrm{T}} \sqrt{\mathcal{S}}=O\left(\eta^{2}\right)$.

(with a mass $M$ and a stiffness $K$ ) satisfies $M \ddot{X}=K\left(u_{x}-X\right)$ where $u_{x}$ is the horizontal surface displacement. In the harmonic regime it follows that $X=\frac{\omega_{0}^{2}}{\omega_{0}^{2}-\omega^{2}} u_{x}\left(\omega_{0}^{2}=\frac{K}{M}\right)$. In the effective medium approach, for a wavelength much greater than the array spacing the average force per unit surface is identified to the normal stress along $x$ hence $\sigma_{x z}=\frac{K}{\mathcal{S}}\left(X-u_{x}\right)(\mathcal{S}$ is the surface area of the unit cell), resulting in

$$
\sigma_{\alpha z}=Z_{\alpha} u_{\alpha}, \quad Z_{\alpha}=\frac{K}{\mathcal{S}} \frac{\omega^{2}}{\omega_{0}^{2}-\omega^{2}} .
$$

2 If the physics of the substrate is accounted for, the dynamics of the resonators is considerably simplified. The complexity of actual beam resonators has been accounted for in more recent studies [5, 6, 7, 8, 9, 10, 11, 12]. The case of a plate substrate is simpler than that of a half-plane substrate, both experimentally and theoretically. From a theoretical point of view, this is because the dynamics of the resonator and of the substrate can be reduced to a one-dimensional problem. Besides a rigid plate substrate does not couple easily to the flexural motions of the resonators, which further simplifies the analysis to the coupling between the flexural modes in the plate and the longitudinal modes in the resonators [5, 6]. The influence of flexural modes in the resonators for less rigid plates has been studied theoretically in [7] and recently confirmed experimentally [10, 11].

The first experiments on the interaction of beams with a half-plane substrate have been reported in [13], and largely developed in the framework of the metaforest project [? ]. From a theoretical point of view, this situation is more involved; the reduction of model to one-dimension is not possible in the substrate and it is not straightforward in the resonator. Besides, the substrate can now couples efficiently to the beams both in flexion and compression; and as long as the full wave problem has not be specified, it is not possible to infer that displacements and $a$ fortiori resonances in one direction can be neglected. In [7] the problem is solved considering the longitudinal resonances only. It is specified that the flexural motion could be accounted for but the analysis seems sufficient for the specific application considered by the authors. However, 
there is a greyness in the term rods or rod-like resonator introduced by the authors and which has been further used e.g. in [8, 12]: beams would support both flexural and longitudinal waves while rods would only support compressional waves. This denomination suggests that the ability of the resonators to couple in flexion or in compression with the substrate is an intrinsic property of the resonator. However, the weak coupling in flexion observed for a plate substrate cannot be attributable to the resonators on their own and the term "rod-like resonators" is a minima confusing. The first study on the effect of flexural resonances has been proposed recently in [9]; the interest is in the derivation of approximate dispersion relations of Rayleigh waves using exotic junction conditions between the substrate and the array of beams. The authors introduce the term "beam-like resonator" which refer to situations where the longitudinal motions could be neglected; as for its "rod-like" version, this term is confusing as long as the wave context has not be specified. Recently, we considered longitudinal and flexural motions of beams and we restricted our analysis to the low frequency regime [1]. Low frequency ment that only flexural resonances can take place namely frequencies below the first longitudinal resonance frequency (see forthcoming figures 2 and 3 ).

The present study aims to generalize the results of [1] to higher frequencies. As in [1], both flexural and longitudinal motions are accounted for and higher frequencies means that longitudinal resonances, in addition to the flexural ones, can take place. We consider beams with circular cross- section in three dimensions. (The case of plates in two-dimensions, much simpler, is given in Appendix B]) The adaptation of the methodology proposed in [1] is demanding. The asymptotic analysis has to account for a separation of the length scales between the periodicity of the array and the beam height which was already the case in [1]; however we have now to account for an additional separation of the scales between the wavelength associated to flexural resonances and that associated to the longitudinal resonances. The resulting full model which is the main result of our study is given in $\$ 2$ and discussed in view of existing models. The derivation of the model based on asymptotic analysis and homogenization is detailed in $\$ 3$ Eventually we provide in $\$ 4$ a validation of the model for a problem of scattering by comparison with direct numerical simulations in a two-dimensional settings.

\section{The actual problem and the effective problem}

We consider an array of beams atop an isotropic elastic substrate in three-dimensions, with $\mathbf{x}=(x, y, z)$, see figure 1 The unit cell of the array has a section $\mathcal{S}=\ell_{x} \times \ell_{y}$; the beams have a circular cross-section with radius $r_{\mathrm{b}}$ and their height is $h_{\mathrm{b}}$ with $h_{\mathrm{b}} \gg r_{\mathrm{b}}$; we define $\varphi=\pi r_{\mathrm{b}}^{2} / \mathcal{S}$. We denote $\left(\lambda_{\mathrm{b}}, \mu_{\mathrm{b}}, \rho_{\mathrm{b}}\right)$ the Lamé coefficients and the mass density of the beams, $\left(\lambda_{\mathrm{s}}, \mu_{\mathrm{s}}, \rho_{\mathrm{s}}\right)$ those of the substrate. We also introduce the Young's modulus $E_{\mathrm{b}}=\frac{\mu_{\mathrm{b}}\left(3 \lambda_{\mathrm{b}}+2 \mu_{\mathrm{b}}\right)}{\lambda_{\mathrm{b}}+\mu_{\mathrm{b}}}$ and Poisson ratio $v_{\mathrm{b}}=\frac{\lambda_{\mathrm{b}}}{2\left(\lambda_{\mathrm{b}}+\mu_{\mathrm{b}}\right)}$ of the beam. We consider a low frequency regime for which the typical wavelength $k$ in the substrate being of the order of magnitude of $k_{\mathrm{T}}=\frac{\omega}{c_{\mathrm{T}}}$ or $k_{\mathrm{L}}=\frac{\omega}{c_{\mathrm{L}}}$ satisfies $k \sqrt{\mathcal{S}} \ll 1$, with $c_{\mathrm{T}}=\sqrt{\frac{\mu_{\mathrm{s}}}{\rho_{\mathrm{s}}}}, c_{\mathrm{L}}=\sqrt{\frac{\lambda_{\mathrm{s}}+2 \mu_{\mathrm{s}}}{\rho_{\mathrm{s}}}}$, with $\omega$ the angular frequency. 


\subsection{The physical problem}

In the actual problem, the equations of elastodynamics apply

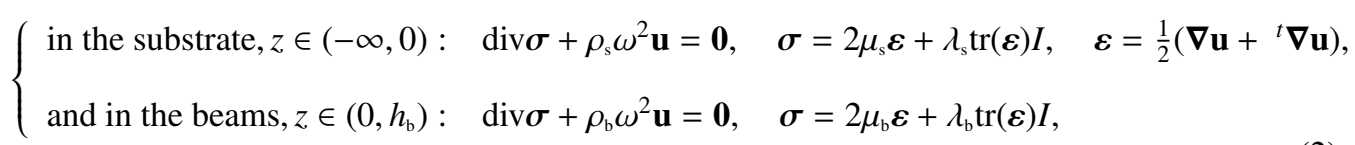

where $\mathbf{u}$ is the displacement vector, $\sigma$ the stress tensor and $\boldsymbol{\varepsilon}$ the strain tensor; $I$ stands for the identity matrix. At each interface between an elastic medium (the beams or the substrate) and air, 61 the stress free condition $\boldsymbol{\sigma} \mathbf{n}=\mathbf{0}$ applies (with $\mathbf{n}$ the normal to the interface). At each interface 62 between the beams and the substrate, the displacement and the normal stress are continuous.

${ }_{63}$ This problem can be solved once the source $\mathbf{u}^{\text {inc }}$ has been defined and accounting for the radiation condition for $z \rightarrow-\infty$ which applies to the scattered field $\left(\mathbf{u}-\mathbf{u}^{\text {inc }}\right)$.

\section{2.2. The effective problem}

Below we provide the main results of the analysis developed in the forthcoming $\$ 3$ It will be shown that the problem reduces to a much simpler one set on the substrate on its own and where the effect of the array is reduced to impedance conditions. Specifically, we obtain that the effective problem reads

$$
\left\{\begin{array}{l}
\operatorname{div} \boldsymbol{\sigma}+\rho_{\mathrm{s}} \omega^{2} \mathbf{u}=\mathbf{0}, \quad \boldsymbol{\sigma}=2 \mu_{\mathrm{s}} \boldsymbol{\varepsilon}+\lambda_{\mathrm{s}} \operatorname{tr}(\boldsymbol{\varepsilon}) I, \quad \text { for } z \in(-\infty, 0), \\
\sigma_{a z}\left(\mathbf{x}^{\prime}, 0\right)=\mu_{\mathrm{s}} k_{\mathrm{T}} f_{\mathrm{F}}\left(\omega, h_{\mathrm{b}}\right) u_{a}\left(\mathbf{x}^{\prime}, 0\right), \quad a=x, y, \quad \sigma_{z z}\left(\mathbf{x}^{\prime}, 0\right)=\mu_{\mathrm{s}} k_{\mathrm{T}} f_{\mathrm{L}}\left(\omega, h_{\mathrm{b}}\right) u_{z}\left(\mathbf{x}^{\prime}, 0\right),
\end{array}\right.
$$

where $\mathbf{x}^{\prime}=(x, y)$ and

$$
\begin{cases}f_{\mathrm{F}}\left(\omega, h_{\mathrm{b}}\right)=\varphi \frac{\rho_{\mathrm{b}}}{\rho_{\mathrm{s}}} \frac{k_{\mathrm{T}}}{\kappa} \frac{\operatorname{sh} \kappa h_{\mathrm{b}} \cos \kappa h_{\mathrm{b}}+\operatorname{ch} \kappa h_{\mathrm{b}} \sin \kappa h_{\mathrm{b}}}{1+\cos \kappa h_{\mathrm{b}} \operatorname{ch} \kappa h_{\mathrm{b}}}, & \kappa=\left(\frac{4 \rho_{\mathrm{b}} \omega^{2}}{E_{\mathrm{b}} r_{\mathrm{b}}^{2}}\right)^{1 / 4}, \\ f_{\mathrm{L}}\left(\omega, h_{\mathrm{b}}\right)=\varphi \frac{\rho_{\mathrm{b}}}{\rho_{\mathrm{s}}} \frac{k_{\mathrm{T}}}{K} \tan K h_{\mathrm{b}}, & K=\sqrt{\frac{\rho_{\mathrm{b}}}{E_{\mathrm{b}}}} \omega .\end{cases}
$$

The boundary conditions on $\sigma_{a z}, a=x, y$, are the same as in [1], the impedance function $f_{\mathrm{F}}\left(\omega, h_{\mathrm{b}}\right)$ encapsulating the flexural resonances at roughly $\kappa h_{\mathrm{b}} \sim \frac{\pi}{2}+n \pi, n$ integer ${ }^{1}$. Next, the impedance function $f_{\mathrm{L}}\left(\omega, h_{\mathrm{b}}\right)$ encapsulates the longitudinal resonances at $K h_{\mathrm{b}}=\frac{\pi}{2}+n \pi, n$ integer, and the first flexural resonance frequency $\omega_{\mathrm{F}}$ is much smaller than the first longitudinal resonance $\omega_{\mathrm{L}}$ with $\omega_{\mathrm{L}} \sim \frac{h_{\mathrm{b}}}{r_{\mathrm{b}}} \omega_{\mathrm{F}} \gg \omega_{\mathrm{F}}$. This is illustrated in figure 2 where we report typical variations of the impedance functions against $h_{\mathrm{b}}$. Note that in [1], the analysis holds for small height $h_{\mathrm{b}}$ resulting in a linearized version of $f_{\mathrm{L}}$ whose validity has been inspected up to $h_{\mathrm{b}}=30 \mathrm{~m}$, see figure 7 in [1] as (3) simplifies to $\sigma_{z z}\left(\mathbf{x}^{\prime}, 0\right)=\varphi \rho_{\mathrm{b}} h_{\mathrm{b}} \omega^{2} u_{z}\left(\mathbf{x}^{\prime}, 0\right)$. For $h_{\mathrm{b}} \rightarrow 0$ which corresponds to roughnesses, we get $\sigma_{i z}\left(\mathbf{x}^{\prime}, 0\right)=\varphi \rho_{\mathrm{b}} h_{\mathrm{b}} \omega^{2} u_{i}\left(\mathbf{x}^{\prime}, 0\right), i=x, y, z$, and in this limit the substrate couples to the array equally in flexion and compression by a term of added mass.

${ }^{1}$ The dispersion relation of the flexural resonances reads $D(\kappa)=\left(1+\cos \kappa h_{\mathrm{b}} \operatorname{ch} \kappa h_{\mathrm{b}}\right)=0$ resulting in $\kappa h_{\mathrm{b}}=1.875$, $4.694,7.855,10.995, \cdots$; the approximation $\kappa h_{\mathrm{b}} \sim \frac{\pi}{2}+n \pi$ provides $\kappa h_{\mathrm{b}}=1.571,4.712,7.854,10.995 \cdots$ and the agreement is better as $n$ increases (with $D(\kappa)=1$ ). 


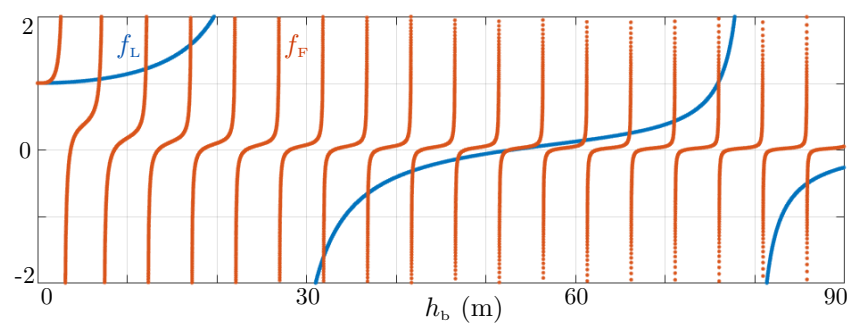

Figure 2: Variations of the impedance functions $f_{\mathrm{F}}$ and $f_{\mathrm{L}}$ in 4 against the beam height $h_{\mathrm{b}}$. The flexural resonances correspond to $\left|f_{\mathrm{F}}\right| \rightarrow \infty$ and the longitudinal resonance to $\left|f_{\mathrm{L}}\right| \rightarrow \infty$

Once the effective problem (3) in the substrate has been resolved, the solution in the region of the beams can be post-processed. Indeed, the asymptotic analysis which is detailed in section 3 shows that the vertical displacement $U_{z}$ in the region of the beams is solution to

$$
\begin{cases}\frac{\partial^{2} U_{z}}{\partial z^{2}}+K^{2} U_{z}=0, & \text { with } K=\sqrt{\frac{\rho_{\mathrm{b}}}{E_{\mathrm{b}}}} \omega \\ U_{z}\left(\mathbf{x}^{\prime}, 0\right)=u_{z}\left(\mathbf{x}^{\prime}, 0\right), & \frac{\partial U_{z}}{\partial z}\left(\mathbf{x}^{\prime}, h_{\mathrm{b}}\right)=0\end{cases}
$$

and the horizontal displacements $U_{a}, a=x, y$, are solutions to Euler-Bernouilli problems

$$
\begin{cases}\frac{\partial^{4} U_{a}}{\partial z^{4}}-\kappa^{4} U_{a}=0, & \text { with } \kappa=\left(\frac{4 \rho_{\mathrm{b}} \omega^{2}}{E_{\mathrm{b}} r_{\mathrm{b}}^{2}}\right)^{1 / 4}, \\ U_{a}\left(\mathbf{x}^{\prime}, 0\right)=u_{a}\left(\mathbf{x}^{\prime}, 0\right), & \frac{\partial U_{a}}{\partial z}\left(\mathbf{x}^{\prime}, 0\right)=0, \quad \frac{\partial^{2} U_{a}}{\partial z^{2}}\left(\mathbf{x}^{\prime}, h_{\mathrm{b}}\right)=\frac{\partial^{3} U_{a}}{\partial z^{3}}\left(\mathbf{x}^{\prime}, h_{\mathrm{b}}\right)=0 .\end{cases}
$$

In (5)-(6), boundary conditions at the top of the beams $z=h_{\mathrm{b}}$ correspond to free-end boundary conditions while at $z=0$, we have a clamped boundary condition, meaning prescribed displacements at the junction with the substrate and zero rotation. Solving these two problems for which the inputs are the prescribed displacements $\mathbf{u}\left(\mathbf{x}^{\prime}, 0\right)$ at the junction with the substrate gives

$$
\left\{\begin{aligned}
U_{a}(\mathbf{x})= & u_{a}\left(\mathbf{x}^{\prime}, 0\right) V_{\mathrm{F}}(z), \quad a=x, y, \quad U_{z}(\mathbf{x})=u_{z}\left(\mathbf{x}^{\prime}, 0\right) V_{\mathrm{L}}(z), \\
\text { with } \quad V_{\mathrm{L}}(z) & =\cos (K z)+\tan \left(K h_{\mathrm{b}}\right) \sin (K z), \\
& V_{\mathrm{F}}(z)=a\left(\operatorname{ch} \kappa\left(z-h_{\mathrm{b}}\right)+\cos \kappa\left(z-h_{\mathrm{b}}\right)\right)+b\left(\operatorname{sh} \kappa\left(z-h_{\mathrm{b}}\right)+\sin \left(z-h_{\mathrm{b}}\right)\right),
\end{aligned}\right.
$$

76 with $a=\frac{1}{d}\left(\operatorname{ch} \kappa h_{\mathrm{b}}+\cos \kappa h_{\mathrm{b}}\right), b=\frac{1}{d}\left(\operatorname{sh} \kappa h_{\mathrm{b}}-\sin \kappa h_{\mathrm{b}}\right), d=2\left(1+\operatorname{ch} \kappa h_{\mathrm{b}} \cos \kappa h_{\mathrm{b}}\right)$. It is worth noting that at the dominant order, the effective surface is isotropic even for different spacings $\ell_{x}$ along $x$ and $\ell_{y}$ along $y$.

\section{2.3. Comparison with previous models}

If the flexural motions are neglected, our impedance conditions (3) on $\sigma_{z z}$ and the longitudinal displacements $U_{z}$ in the region of the beams (7) are consistent with the findings of [5, 6]. As previously said, in these references, the flexural motions are disregarded since the beams are connected to a rigid plate substrate. In the present case, for a half-space substrate, we shall see 
in $\$ 4$ that neglecting the flexural resonances leads to incorrect predictions. Next, two recent studies have accounted for flexural resonances only [1, 9]. In [1], this is justified by the low frequency regime below the first longitudinal resonance and our present model includes this case, as previously stated. The comparison with the study [9] is made difficult by the fact that the authors consider different junction conditions among which some are exotic, but the case of vertical beams clamped to a substrate $\left(U_{a}\left(\mathbf{x}^{\prime}, 0\right)=u_{a}\left(\mathbf{x}^{\prime}, 0\right), \frac{\partial U_{a}}{\partial z}\left(\mathbf{x}^{\prime}, 0\right)=0\right.$ from (6) have not been considered. To be specific, 3 junction conditions have been considered in [9], called simply supported, beams on a rail and fully matched. They read, for $a=x, y$,

$$
\left\{\begin{array}{lll}
\text { simply supported } & U_{a}\left(\mathbf{x}^{\prime}, 0\right)=u_{a}\left(\mathbf{x}^{\prime}, 0\right), & \frac{\partial^{2} U_{a}}{\partial z^{2}}\left(\mathbf{x}^{\prime}, 0\right)=0, \\
\text { beams on the rails, }, & \frac{\partial^{2} U_{a}}{\partial z^{2}}\left(\mathbf{x}^{\prime}, 0\right)=0, & \frac{\partial U_{a}}{\partial z}\left(\mathbf{x}^{\prime}, 0\right)=-\frac{\partial u_{z}}{\partial a}\left(\mathbf{x}^{\prime}, 0\right), \\
\text { fully matched }, & U_{a}\left(\mathbf{x}^{\prime}, 0\right)=u_{a}\left(\mathbf{x}^{\prime}, 0\right), & \frac{\partial U_{a}}{\partial z}\left(\mathbf{x}^{\prime}, 0\right)=-\frac{\partial u_{z}}{\partial a}\left(\mathbf{x}^{\prime}, 0\right) .
\end{array}\right.
$$

\section{Asymptotic analysis}

The analysis is conducted assuming low frequencies which means that the typical array spacing $\ell$, with $\mathcal{S}=\ell_{x} \ell_{y}=\ell^{2}$, is small compared to the typical incident wavelength $1 / k$, hence $k \ell \ll 1$ (with for instance $k=k_{\mathrm{T}}$ ). This ensures that incoming waves perceive the interface at $z=0$ essentially as an effective homogeneous interface. Next, in our analysis, we do not impose particular scalings on the material properties. We do not assume neither a sparse array of beams which means that $r_{\mathrm{b}}=O(\ell)$ hence $\varphi=O(1)$. However we cannot dispense with a careful length scalings in the vertical direction. Indeed, and as previously said, flexural and longitudinal resonances of beams are associated with different length scales

$$
h_{\mathrm{F}}=\kappa^{-1} \quad \text { and } h_{\mathrm{L}}=K^{-1},
$$

(with $\kappa$ and $K$ given in (4)) as illustrated with the typical deformation shown in figure 3 . From (4), we have $k h_{\mathrm{L}}=O(1)$ while $k h_{\mathrm{F}}=O(\sqrt{k \ell}) \ll 1$. It results a hierarchy of 3 scales $\ell \ll h_{\mathrm{F}} \ll h_{\mathrm{L}}$ which is accounted for owing to a small parameter $\eta$ such that

$$
k \ell=\eta^{2}, \quad k h_{\mathrm{F}}=O(\eta), \quad \text { and } \quad k h_{\mathrm{b}}=O(1) .
$$

Accordingly, with $\mathbf{x}=(x, y, z)$, the analysis will be conducted owing to the rescaled spatial coordinates being

$$
\tilde{z}=\frac{z}{\eta}, \quad(\hat{x}, \hat{y}, \hat{z})=\frac{(x, y, z)}{\eta^{2}} .
$$




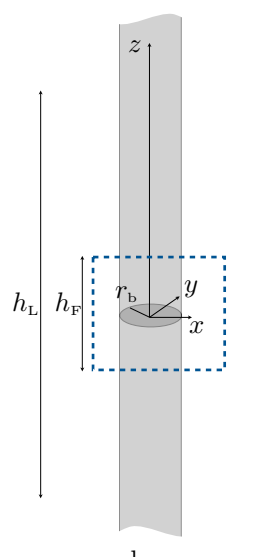

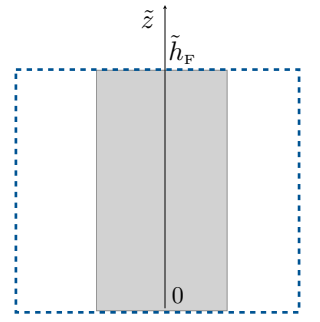

vertical rescaling

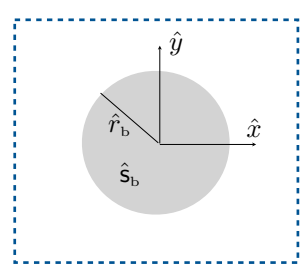

cross-sectional rescaling

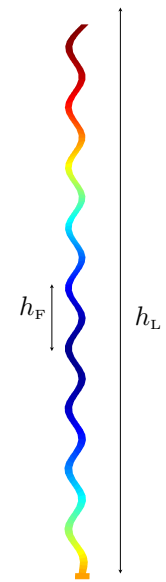

Figure 3: The two length scales in the vertical direction, with $h_{\mathrm{L}} \sim h_{\mathrm{b}}$ the length scale associated with longitudinal resonances and $h_{\mathrm{F}} \ll h_{\mathrm{b}}$ the length scale associated with flexural resonances; the center panels show the vertical rescaling with the $\tilde{z}$-coordinate, $\tilde{z} \in\left(0, \tilde{h}_{\mathrm{F}}\right)$ and the horizontal rescaling with $\hat{\mathbf{x}}^{\prime}=(\hat{x}, \hat{y}) \in \hat{\mathrm{s}}_{\mathrm{b}}$. The right panel show a typical deformation associated to the displacement $U_{x}$ with variations at the scale $h_{\mathrm{F}}$ and colors associated to $U_{z}$ with variations at the scale $h_{\mathrm{L}}$

(We define $\hat{\mathbf{x}}=(\hat{x}, \hat{y}, \hat{z})$ and $\hat{\mathbf{x}}^{\prime}=(\hat{x}, \hat{y})$.) The coordinate $\tilde{z}$ aims to capture the rapid (in $\frac{1}{\eta}$ ) spatial variations relative to flexural motions, while $\hat{\mathbf{x}}$ aim to capture the very rapid (in $\frac{1}{\eta^{2}}$ ) spatial variations relative to evanescent fields. Eventually the coordinate $\mathbf{x}$ describe the slow variations (in $O(1)$ ) associated with the wave propagation. Associated to these coordinates, rescaled lengths are defined

$$
\left(\tilde{h}_{\mathrm{F}}, \hat{r}_{\mathrm{b}}, \hat{\ell}_{x}, \hat{\ell}_{y}\right)=\left(\frac{h_{\mathrm{F}}}{\eta}, \frac{r_{\mathrm{b}}}{\eta^{2}}, \frac{\ell_{x}}{\eta^{2}}, \frac{\ell_{y}}{\eta^{2}}\right) .
$$

\subsection{Effective wave equation in the region of the beams}

89 3.1.1. Notations

In the region of the array of beams, the displacements and the stresses vary in the horizontal direction over small distances dictated by $\ell\left(\ell=\sqrt{\ell_{x} \ell_{y}}\right)$ and over large distances dictated by the incoming waves being of the same order of magnitude than $h_{\mathrm{b}}$. These two scales are accounted for by the coordinates $\mathbf{x}^{\prime}=(x, y)$ and $\hat{\mathbf{x}}^{\prime}=(\hat{x}, \hat{y})$ with $\hat{\mathbf{x}}^{\prime}=\frac{\mathbf{x}^{\prime}}{\eta^{2}}$; we define $\hat{\mathbf{s}}_{\mathrm{b}}$ the cross-section of a beam and

$$
\hat{\mathbf{x}}^{\prime} \in \hat{\mathrm{S}}_{\mathrm{b}}=\left\{\left|\hat{\mathbf{x}}^{\prime}\right| \in\left(0, \hat{r}_{\mathrm{b}}\right)\right\} .
$$

In the vertical direction, the displacements and the stresses vary over the intermediate distance $h_{\mathrm{F}}$ that we expect to play a role for the flexural motions only and over $h_{\mathrm{b}}$; this is accounted for by the rescaled coordinates $z$ and $\tilde{z}$, with

$$
\tilde{z} \in\left(0, \tilde{h}_{\mathrm{F}}\right)
$$

It follows that the fields $(\mathbf{u}, \boldsymbol{\sigma})$ are written of the form

$$
\mathbf{u}=\sum_{n \geq 0} \eta^{n} \mathbf{V}^{n}\left(\mathbf{x}, \hat{\mathbf{x}}^{\prime}, \tilde{z}\right), \quad \boldsymbol{\sigma}=\sum_{n \geq 0} \eta^{n} \mathbf{\Sigma}^{n}\left(\mathbf{x}, \hat{\mathbf{x}}^{\prime}, \tilde{z}\right),
$$


90 with all the terms in the expansions being periodic with respect to $\tilde{z} \in\left(0, \tilde{h}_{\mathrm{F}}\right)$ (figure ??).

In the asymptotic analysis, the small scale $\hat{\mathbf{x}}^{\prime}$ aims to disappear since we are interested in macroscopic fields. Hence, we define at each order $n$ the macroscopic displacement $U_{z}^{n}$ and axial stress

$$
U_{z}^{n}=\frac{1}{\hat{\mathcal{S}}_{\mathrm{b}}} \int_{\hat{\mathrm{s}}_{\mathrm{b}}} V_{z}^{n} \mathrm{~d} \hat{\mathbf{x}}^{\prime}, \quad N_{z z}^{n}=\int_{\hat{\mathrm{s}}_{\mathrm{b}}} \Sigma_{z z}^{n} \mathrm{~d} \hat{\mathbf{x}}^{\prime},
$$

where $\hat{\mathcal{S}}_{\mathrm{b}}=\left|\hat{\mathrm{S}}_{\mathrm{b}}\right|=\pi \hat{r}_{\mathrm{b}}^{2}$ is the rescaled surface area of beam section. We also define the macroscopic displacements, shear stresses and bending moments

$$
U_{a}^{n}=\frac{1}{\hat{\mathcal{S}}_{\mathrm{b}}} \int_{\hat{\mathrm{s}}_{\mathrm{b}}} V_{a}^{n} \mathrm{~d} \hat{\mathbf{x}}^{\prime}, \quad T_{a}^{n}=\int_{\hat{\mathrm{s}}_{\mathrm{b}}} \Sigma_{a z}^{n} \mathrm{~d} \hat{\mathbf{x}}^{\prime}, \quad a=x, y, \quad M_{x}^{n}=-\int_{\hat{\mathrm{s}}_{\mathrm{b}}} \hat{x} \Sigma_{z z}^{n} \mathrm{~d} \hat{\mathbf{x}}^{\prime}, \quad M_{y}^{n}=-\int_{\hat{\mathrm{s}_{\mathrm{b}}}} \hat{y} \Sigma_{z z}^{n} \mathrm{~d} \hat{\mathbf{x}}^{\prime} .
$$

\section{3.1.2. The problem in the beam}

According to the expansions in (10) the differential operator reads

$$
\boldsymbol{\nabla} \rightarrow \frac{1}{\eta^{2}} \boldsymbol{\nabla}_{\hat{\mathbf{x}}^{\prime}}+\frac{\mathbf{e}_{z}}{\eta} \frac{\partial}{\partial \tilde{z}}+\boldsymbol{\nabla}_{\mathbf{x}},
$$

where $\mathbf{e}_{z}=(0,0,1)$. Hence, the system in the region of the beams reads, from (2),

$$
\left\{\begin{array}{l}
\left(\mathrm{E}_{a}\right)^{n} \quad\left(\frac{\partial \Sigma_{a x}^{n+2}}{\partial \hat{x}}+\frac{\partial \Sigma_{a y}^{n+2}}{\partial \hat{y}}\right)+\frac{\partial \Sigma_{a z}^{n+1}}{\partial \tilde{z}}+\frac{\partial \Sigma_{a x}^{n}}{\partial x}+\frac{\partial \Sigma_{y a}^{n}}{\partial y}+\frac{\partial \Sigma_{a z}^{n}}{\partial z}+\rho_{\mathrm{b}} \omega^{2} V_{a}^{n}=0, \quad a=x, y, \\
\left(\mathrm{E}_{z}\right)^{n} \quad\left(\frac{\partial \Sigma_{x z}^{n+2}}{\partial \hat{x}}+\frac{\partial \Sigma_{y z}^{n+2}}{\partial \hat{y}}\right)+\frac{\partial \Sigma_{z z}^{n+1}}{\partial \tilde{z}}+\frac{\partial \Sigma_{x z}^{n}}{\partial x}+\frac{\partial \Sigma_{y z}^{n}}{\partial y}+\frac{\partial \Sigma_{z z}^{n}}{\partial z}+\rho_{\mathrm{b}} \omega^{2} V_{z}^{n}=0, \\
(\mathrm{C})^{n} \quad \mathbf{\Sigma}^{n}=\mathrm{A} \boldsymbol{\varepsilon}^{\hat{\mathbf{x}}^{\prime}}\left(\mathbf{V}^{n+2}\right)+\mathrm{A} \boldsymbol{\varepsilon}^{\tilde{z}}\left(\mathbf{V}^{n+1}\right)+\mathrm{A} \boldsymbol{\varepsilon}^{\mathbf{x}}\left(\mathbf{V}^{n}\right),
\end{array}\right.
$$

where we have implicitly that $\boldsymbol{\Sigma}^{m}=\mathbf{V}^{m}=0$ for $m<0$ and where $\mathrm{A} \boldsymbol{\varepsilon}(V)=2 \mu_{\mathrm{b}} \boldsymbol{\varepsilon}(V)+\lambda_{\mathrm{b}} \operatorname{tr}(\boldsymbol{\varepsilon}(V)) I$ which holds for $\varepsilon=\varepsilon^{\hat{x}^{\prime}}, \varepsilon=\varepsilon^{\tilde{z}}$ and $\varepsilon=\varepsilon^{\mathbf{x}}$. We shall also use the stress-strain relation written in the form

$$
\left(\mathrm{C}^{\prime}\right)^{n} \quad \varepsilon^{\hat{\mathbf{x}}^{\prime}}\left(\mathbf{V}^{n+2}\right)+\varepsilon^{\tilde{z}}\left(\mathbf{V}^{n+1}\right)+\varepsilon^{\mathbf{x}}\left(\mathbf{V}^{n}\right)=\frac{1+\nu_{\mathrm{b}}}{E_{\mathrm{b}}} \mathbf{\Sigma}^{n}-\frac{\nu_{\mathrm{b}}}{E_{\mathrm{b}}} \operatorname{tr}\left(\mathbf{\Sigma}^{n}\right) I .
$$

The symmetric strain tensors with respect to $\hat{\mathbf{x}}^{\prime}, \tilde{z}$ and $\mathbf{x}$ have the following forms

$$
\begin{aligned}
\varepsilon^{\hat{\mathbf{x}}^{\prime}}(\mathbf{V}) & =\frac{1}{2}\left(\begin{array}{ccc}
2 \frac{\partial V_{x}}{\partial \hat{x}} & \frac{\partial V_{x}}{\partial \hat{y}}+\frac{\partial V_{y}}{\partial \hat{x}} & \frac{\partial V_{z}}{\partial \hat{x}} \\
2 \frac{\partial V_{y}}{\partial \hat{y}} & \frac{\partial V_{z}}{\partial \hat{y}}
\end{array}\right), \quad \varepsilon^{\tilde{z}}(\mathbf{V})=\frac{1}{2}\left(\begin{array}{ccc}
0 & 0 & \frac{\partial V_{x}}{\partial \tilde{z}} \\
0 & \frac{\partial V_{y}}{\partial \tilde{z}} \\
& 2 \frac{\partial V_{z}}{\partial \tilde{z}}
\end{array}\right), \\
\varepsilon^{\mathbf{x}}(\mathbf{V}) & =\frac{1}{2}\left(\begin{array}{ccc}
2 \frac{\partial V_{x}}{\partial x} & \frac{\partial V_{x}}{\partial y}+\frac{\partial V_{y}}{\partial x} & \frac{\partial V_{x}}{\partial z}+\frac{\partial V_{z}}{\partial x} \\
2 \frac{\partial V_{y}}{\partial y} & \frac{\partial V_{y}}{\partial z}+\frac{\partial V_{z}}{\partial y} \\
2 \frac{\partial V_{z}}{\partial z}
\end{array}\right) .
\end{aligned}
$$

92 From now on, we shall use the hierarchy of equations in (13) and (14). 


\subsubsection{Useful forms of the displacements and stresses}

We start by providing the explicit dependence with respect to the microscopic coordinate $\hat{\mathbf{x}}^{\prime}$ of the displacements $\left(\mathbf{V}^{0}, \mathbf{V}^{1}\right)$ and the of stress $\Sigma^{0}$.

- Displacements at orders 0 and 1 - The dependance of $\mathbf{V}^{0}$ and $\mathbf{V}^{1}$ on $\hat{\mathbf{x}}^{\prime}$ read

$$
\left\{\begin{array}{llrl}
V_{x}^{0} & =U_{x}^{0}\left(\mathbf{x}^{\prime}, \tilde{z}\right), & V_{y}^{0}=U_{y}^{0}\left(\mathbf{x}^{\prime}, \tilde{z}\right), & V_{z}^{0}=U_{z}^{0}(\mathbf{x}), \\
V_{x}^{1}=U_{x}^{1}(\mathbf{x}, \tilde{z})+\Omega^{1}(\mathbf{x}) \hat{y}, & V_{y}^{1}=U_{y}^{1}(\mathbf{x}, \tilde{z})-\Omega^{1}(\mathbf{x}) \hat{x}, & V_{z}^{1}=U_{z}^{1}(\mathbf{x})-\frac{\partial}{\partial \tilde{z}}\left(U_{x}^{0} \hat{x}+U_{y}^{0} \hat{y}\right) .
\end{array}\right.
$$

To get (15), we have used $\left(\mathrm{C}^{\prime}\right)^{-2}$ and $\left(\mathrm{C}^{\prime}\right)^{-1}$ in $(14)$ from which $\varepsilon^{\hat{\mathbf{x}}^{\prime}}\left(\mathbf{V}^{0}\right)=\mathbf{0}$ and $\varepsilon^{\hat{\mathbf{x}}^{\prime}}\left(\mathbf{V}^{1}\right)+\varepsilon^{\tilde{z}}\left(\mathbf{V}^{0}\right)=$ 0. It follows that $\left(V_{x}^{m}, V_{y}^{m}\right), m=0,1$, are associated with rigid body motions (translation and rotation), namely $V_{x}^{m}=U_{x}^{m}(\mathbf{x}, \tilde{z})+\Omega^{m}(\mathbf{x}, \tilde{z}) \hat{y}, V_{y}^{m}=U_{y}^{m}(\mathbf{x}, \tilde{z})-\Omega^{m}(\mathbf{x}, \tilde{z}) \hat{x}$. We also get that $V_{z}^{0}$ depends only on $\mathbf{x}$, and that $\frac{\partial}{\partial \hat{a}} V_{z}^{1}=-\frac{\partial}{\partial z} V_{a}^{0}$ for $a=x, y$. Doing so, we obtain the forms reported in (A.2)- A.3. To obtain the forms given in (15), we have anticipated the following properties that we shall proof in the Appendix A.

$$
\text { To be shown : } \quad P_{1}: \frac{\partial U_{z}^{1}}{\partial \tilde{z}}=0 \quad P_{2}: \frac{\partial \Omega^{1}}{\partial \tilde{z}}=0, \quad P_{3}: \Omega^{0}=0, \quad P_{4}: \frac{\partial U_{a}^{0}}{\partial z}=0, \quad a=x, y \text {. }
$$

- Stress at order 0 - The dependence of the stress $\boldsymbol{\Sigma}^{0}$ on $\hat{\mathbf{x}}^{\prime}$ can be obtained explicitly too. Indeed, from $\left(\mathrm{E}_{a}\right)^{-2},\left(\mathrm{E}_{z}\right)^{-2}$ and from $(\mathrm{C})^{0}$ in $(13)$, we have $\operatorname{div}_{\hat{\mathbf{x}}^{\prime}} \boldsymbol{\Sigma}^{0}=\mathbf{0}, \boldsymbol{\Sigma}^{0}=\mathrm{A} \boldsymbol{\varepsilon}^{\hat{\mathbf{x}}^{\prime}}\left(\mathbf{V}^{2}\right)+\mathrm{A} \boldsymbol{\varepsilon}^{\tilde{z}}\left(\mathbf{V}^{1}\right)+$ $A \varepsilon^{\mathbf{x}}\left(\mathbf{V}^{0}\right)$. As $\left(\mathbf{V}^{0}, \mathbf{V}^{1}\right)$ are known from $(15)$, the problem is set on $\left(\boldsymbol{\Sigma}^{0}, \mathbf{V}^{2}\right)$ for $\hat{\mathbf{x}}^{\prime} \in \hat{\mathbf{S}}_{\mathrm{b}}$. Besides it can be decomposed into 2 decoupled boundary value problems. The first is set on the horizontal displacements $\left(V_{x}^{2}, V_{y}^{2}\right)$ and the horizontal stress tensor $\Sigma_{a b}^{0}, a, b=x, y$. The second is set on the vertical displacement $V_{z}^{2}$ and the antiplane stress vector $\left(\Sigma_{x z}^{0}, \Sigma_{y z}^{0}\right)$. Both problems can be solved explicitly resulting in

$$
\Sigma_{x x}^{0}=\Sigma_{x y}^{0}=\Sigma_{y y}^{0}=\Sigma_{x z}^{0}=\Sigma_{y z}^{0}=0, \quad \Sigma_{z z}^{0}=E_{\mathrm{b}}\left(\frac{\partial U_{z}^{0}}{\partial z}-\frac{\partial^{2}}{\partial \tilde{z}^{2}}\left(U_{x}^{0} \hat{x}+U_{y}^{0} \hat{y}\right)\right) .
$$

96 The associated displacement $\mathbf{V}^{2}$ that we dont need in the following is given in A.4.

\section{3.1.4. Macroscopic relations for the longitudinal motions}

Once the dependance on $\hat{\mathbf{x}}^{\prime}$ of the displacements and the stress at the order 0 are known, we can determine the macroscopic relations. We start with those associated to the longitudinal motions. The axial stress $N_{z z}^{0}(\mathbf{x})$ in $(11)$ is obtained by integration of $\Sigma_{z z}^{0}$ in (17) over $\hat{\mathbf{x}}^{\prime} \in \hat{\mathrm{s}}_{\mathrm{b}}$ and owing to $\int_{\hat{\mathrm{s}}_{\mathrm{b}}} \hat{x} \mathrm{~d} \hat{\mathbf{x}}^{\prime}=\int_{\hat{\mathrm{s}}_{\mathrm{b}}} \hat{y} \mathrm{~d} \hat{\mathbf{x}}^{\prime}=0$ for a circular cross-section. The equilibrium is then determined using $\left(\mathrm{E}_{z}\right)^{0}$ in (13) which reads $\frac{\partial}{\partial \hat{x}} \Sigma_{x z}^{2}+\frac{\partial}{\partial \hat{y}} \Sigma_{y z}^{2}+\frac{\partial}{\partial \tilde{z}} \Sigma_{z z}^{1}+\frac{\partial}{\partial z} \Sigma_{z z}^{0}+\rho_{\mathrm{b}} \omega^{2} U_{z}^{0}=0$ (we have used that $\Sigma_{x z}^{0}=\Sigma_{y z}^{0}=0$ from (17) and $V_{z}^{0}=U_{z}^{0}(\mathbf{x})$ from (15). Integrating the equilibrium over $\hat{\mathbf{x}}^{\prime} \in \hat{\mathrm{s}}_{\mathrm{b}}$ and accounting for the boundary condition $\Sigma_{x z}^{2} n_{x}+\Sigma_{y z}^{2} n_{y}=0$ on $\partial \hat{\mathrm{s}}_{\mathrm{b}}$, we eventually get

$$
N_{z z}^{0}(\mathbf{x})=E_{\mathrm{b}} \hat{\mathcal{S}}_{\mathrm{b}} \frac{\partial U_{z}^{0}}{\partial z}(\mathbf{x}), \quad \frac{\partial N_{z z}^{0}}{\partial z}(\mathbf{x})+\rho_{\mathrm{b}} \omega^{2} \hat{\mathcal{S}}_{\mathrm{b}} U_{z}^{0}(\mathbf{x})=0
$$

To get the equilibrium, we have used that $\frac{\partial}{\partial \tilde{z}} \int_{\hat{\mathrm{s}}_{\mathrm{b}}} \Sigma_{z z}^{1} \mathrm{~d} \hat{\mathbf{x}}^{\prime}=\frac{\partial}{\partial \tilde{z}} N_{z z}^{1}=0$. Indeed, we have $\frac{\partial}{\partial \tilde{z}} N_{z z}^{1}=$ $-\left(\frac{\partial}{\partial z} N_{z z}^{0}(\mathbf{x})+\rho_{\mathrm{b}} \omega^{2} \varphi U_{z}^{0}(\mathbf{x})\right)$, hence $N_{z z}^{1}$ could be a linear function of $\tilde{z}$. However, such a linear 
dependence is prevented by the periodicity of the field with respect to $\tilde{z}$, which allows us to conclude. It is worth noting that $N_{z z}^{0}$ and $U_{z}^{0}$ do not depend on $\tilde{z}$ associated to flexural motions, as expected. Also, from (18), we recover the wave equation for longitudinal motion

$$
\frac{\partial^{2} U_{z}^{0}}{\partial z^{2}}+K^{2} U_{z}^{0}=0, \quad \text { with } K=\sqrt{\frac{\rho_{\mathrm{b}}}{E_{\mathrm{b}}}} \omega .
$$

\section{3.1.5. Macroscopic relations for the flexural motions}

In this section, we use $a=x, y$. Constitutive relations link the bending moments $M_{a}^{0}$ in (12) to the horizontal displacements $V_{a}^{0}$. They are obtained by integrating $\Sigma_{z z}^{0}$ in (17) over $\hat{\mathbf{x}}^{\prime} \in \hat{\mathrm{s}}_{\mathrm{b}}$ after multiplication by $\hat{a}$. Owing to $\int \hat{x} \mathrm{~d} \hat{\mathbf{x}}^{\prime}=\int \hat{y} \mathrm{~d} \hat{\mathbf{x}}^{\prime}=\int \hat{x} \hat{y} \mathrm{~d} \hat{\mathbf{x}}^{\prime}=0$ and introducing the area moment of inertia $\hat{I}_{\mathrm{b}}=\int \hat{a}^{2} \mathrm{~d} \hat{\mathbf{x}}^{\prime}=\frac{\pi}{4} \hat{r}_{\mathrm{b}}^{4}$, we get

$$
M_{a}^{0}\left(\mathbf{x}^{\prime}, \tilde{z}\right)=E_{\mathrm{b}} \hat{I}_{\mathrm{b}} \frac{\partial^{2} U_{a}^{0}}{\partial \tilde{z}^{2}}\left(\mathbf{x}^{\prime}, \tilde{z}\right), \quad a=x, y .
$$

We now move on the equations of equilibrium. Since $\Sigma_{a z}^{0}=0$ from (17), we also have $T_{a}^{0}=0$ in (12). Next, from $\left(\mathrm{E}_{a}\right)^{0}$ in (13) and using (17), we have $\frac{\partial}{\partial \hat{x}} \Sigma_{x a}^{2}+\frac{\partial}{\partial \hat{y}} \Sigma_{y a}^{2}+\frac{\partial}{\partial \tilde{z}} \Sigma_{z a}^{1}+\rho_{\mathrm{b}} \omega^{2} V_{a}^{0}=0$ that we integrate over $\hat{\mathbf{x}}^{\prime} \in \hat{\mathrm{s}}_{\mathrm{b}}$. Accounting for the boundary condition $\Sigma_{x a}^{2} n_{x}+\Sigma_{y a}^{2} n_{y}=0$ on $\partial \hat{\mathrm{s}}_{\mathrm{b}}$ and for the forms of $V_{a}^{0}$ in [15], we get

$$
T_{a}^{0}=0, \quad \frac{\partial T_{a}^{1}}{\partial \tilde{z}}\left(\mathbf{x}^{\prime}, \tilde{z}\right)+\rho_{\mathrm{b}} \omega^{2} \hat{\mathcal{S}}_{\mathrm{b}} U_{a}^{0}\left(\mathbf{x}^{\prime}, \tilde{z}\right)=0, \quad a=x, y,
$$

(which tells us that $T_{a}^{1}$ does not depend on $z$ ). We still have to determine the relations between bending moments and shear forces. To do so, we use $\left(\mathrm{E}_{z}\right)^{-1}$ in $(13), \frac{\partial}{\partial \hat{x}} \Sigma_{x z}^{1}+\frac{\partial}{\partial \hat{y}} \Sigma_{y z}^{1}+\frac{\partial}{\partial \tilde{z}} \Sigma_{z z}^{0}=0$ that we integrate over $\hat{\mathbf{x}}^{\prime} \in \hat{\mathbf{s}}_{\mathrm{b}}$ after multiplication by $\hat{a}$. Using that $\int_{\hat{\mathrm{s}}_{\mathrm{b}}} \hat{a}\left(\frac{\partial}{\partial \hat{x}} \Sigma_{x z}^{1}+\frac{\partial}{\partial \hat{y}} \Sigma_{y z}^{1}\right) \mathrm{d} \hat{\mathbf{x}}^{\prime}=-T_{a}^{1}$ (integrating by part and accounting for $\Sigma_{x z}^{1} n_{x}+\Sigma_{y z}^{1} n_{y}=0$ on $\partial \hat{s}_{b}$ ), we eventually get

$$
\frac{\partial M_{a}^{0}}{\partial \tilde{z}}\left(\mathbf{x}^{\prime}, \tilde{z}\right)+T_{a}^{1}\left(\mathbf{x}^{\prime}, \tilde{z}\right)=0, \quad a=x, y .
$$

Gathering (20), 21] and (22), we recover the wave equation for the flexural motions

$$
\frac{\partial^{4} U_{a}^{0}}{\partial \tilde{z}^{4}}-\tilde{\kappa}^{4} U_{a}^{0}=0, a=x, y, \quad \text { with } \tilde{\kappa}=\left(\frac{4 \rho_{\mathrm{b}} \omega^{2}}{E_{\mathrm{b}} \hat{r}_{\mathrm{b}}^{2}}\right)^{1 / 4},
$$

which is the rescaled version of (6).

\subsection{Matching conditions and associated boundary/transmission conditions}

The set of beam equations found in the previous section are valid far from the extremities, bottom and top, of the beams. It has to be complemented with boundary conditions. These conditions are obtained by making a suitable asymptotic analysis near the extremities of the beams. Two regimes of boundary layers associated to two different length scales have to be distinguished (see figure 4 ).

The first boundary layer appears when, from the inner region of the beams that we have considered in the previous section, we approach $z=0$ (resp. $z=h_{\mathrm{b}}$ ) within a distance of order $\eta$. By 
rescaling $z$ as $\eta \tilde{z}$ near $z=0$ (resp. $z=\eta \tilde{z}+h_{\mathrm{b}}$ near $\left.z=h_{\mathrm{b}}\right)$ with $\tilde{z}$ lying in $(0,+\infty)(\operatorname{resp} .(-\infty, 0)$ near $z=h_{\mathrm{b}}$ ), we are entering an intermediate region where the fields loose their periodicity in $\tilde{z}$. These mesoscopic regions encapsulate the first boundary layers at the scale $h_{\mathrm{F}}=\kappa^{-1}$.

The second boundary layer appears when, from the mesoscopic regions, we approach $z=0$ (resp. $z=h_{\mathrm{b}}$ ) within a distance of order $\eta^{2}$. Doing so, we entering a microscopic region where evanescent fields enable (i) that the fields in the beam connect those in the soil at $z=0$, (ii) that the fields in the beam feel the traction-free termination at $z=h_{\mathrm{b}}$. These microscopic regions encapsulate the second boundary layers at the scale $\ell$. A consistent dialogue between the microscopic, the mesoscopic and the macroscopic regions is ensured through suitable matching conditions on the displacements and stresses.

\subsubsection{The mesoscopic region near the extremities : beams with boundary layers}

In these intermediate regions where boundary layers for the beams are triggered, new expansions of the fields have to be thought. In particular, we have to drop the periodic assumption on $\tilde{z}$ to encapsulate the boundary layers (periodic terms are typically $\cos \kappa z$ and the loss of periodicity correspond to the appearance of terms in $\cosh \kappa z$ ). Specifically we assume that near $z=z^{*}$ with $z^{*}=0$ or $z^{*}=h_{\mathrm{b}}$, $\mathbf{u}$ and $\sigma$ can be expanded as

$$
\mathbf{u}=\left.\sum_{n \geq 0} \eta^{n} \widetilde{\mathbf{V}}^{n}\right|_{z^{*}}\left(\mathbf{x}^{\prime}, \hat{\mathbf{x}}^{\prime}, \tilde{z}\right), \quad \boldsymbol{\sigma}=\left.\sum_{n \geq 0} \eta^{n} \widetilde{\boldsymbol{\Sigma}}^{n}\right|_{z^{*}}\left(\mathbf{x}^{\prime}, \hat{\mathbf{x}}^{\prime}, \tilde{z}\right), \quad z^{*}=0 \text { or } h_{\mathrm{b}},
$$

where the fast variables $\left(\hat{\mathbf{x}}^{\prime}, \tilde{z}\right)$ lie in $\widetilde{\mathrm{X}}_{0}$ if $z^{*}=0$ and in $\widetilde{\mathrm{X}}_{h_{\mathrm{b}}}$ if $z^{*}=h_{\mathrm{b}}$ with

$$
\widetilde{\mathrm{X}}_{0}=\left\{\hat{\mathbf{x}}^{\prime} \in \hat{\mathrm{s}}_{\mathrm{b}}, \tilde{z} \in(0,+\infty)\right\}, \quad \widetilde{\mathrm{X}}_{h_{\mathrm{b}}}=\left\{\hat{\mathbf{x}}^{\prime} \in \hat{\mathrm{s}}_{\mathrm{b}}, \tilde{z} \in(-\infty, 0)\right\} .
$$

The expansions (24) in the two intermediate regions at the extremities have to match the $\tilde{z}$ periodic expansions $(10)$ inside the beams when $|\tilde{z}|$ goes to infinity. Making asymptotics on this mesoscopic problem is straightforward from the previous section (with $\frac{\partial}{\partial z}=0$, see also A.2 and (A.5) and we get the following results. First, the hierarchies of equations as in (13) and (14) hold. Next, we find that the axial displacement $\widetilde{V}_{z}^{0}$ and the normal force $\widetilde{N}_{z z}^{0}$ do not depend on $\left(\hat{\mathbf{x}}^{\prime}, \tilde{z},\right)$ and $\tilde{z}$ respectively. Using the matching conditions with the inner region of the beam, we get at the bottom of the beams and at the top of the beams

$$
\left.\widetilde{V}_{z}^{0}\right|_{z^{*}}=U_{z}^{0}\left(\mathbf{x}^{\prime}, z^{*}\right),\left.\quad \widetilde{N}_{z z}^{0}\right|_{z^{*}}=N_{z z}^{0}\left(\mathbf{x}^{\prime}, z^{*}\right), \quad a=x, y, z^{*}=0, h_{\mathrm{b}} .
$$

Secondly, the tangential displacement still satisfies the Euler-Bernouilli equations as in the inner region of the beams, the difference being that the equations are now set on a semi-infinite domain as opposed to a periodic domain. Specifically the mesoscopic Euler-Bernouilli equations read at bottom and the top of the beams

$$
\begin{cases}\left.\widetilde{V}_{a}^{0}\right|_{z^{*}}=\left.\widetilde{U}_{a}^{0}\right|_{z^{*}}\left(\mathbf{x}^{\prime}, \tilde{z}\right), & \left.\widetilde{T}_{a}^{0}\right|_{z^{*}}=0,\left.\quad \widetilde{M}_{a}^{0}\right|_{z^{*}}=E_{\mathrm{b}} \hat{I}_{\mathrm{b}} \frac{\left.\partial^{2} \widetilde{U}_{a}^{0}\right|_{z^{*}}}{\partial \widetilde{z}^{2}} \\ \frac{\left.\partial \widetilde{M}_{a}^{0}\right|_{z^{*}}}{\partial \tilde{z}}+\left.\widetilde{T}_{a}^{1}\right|_{z^{*}}=0, & \frac{\left.\partial \widetilde{T}_{a}^{1}\right|_{z^{*}}}{\partial \tilde{z}}+\left.\rho_{\mathrm{b}} \omega^{2} \hat{\mathcal{S}}_{\mathrm{b}} \widetilde{U}_{a}^{0}\right|_{z^{*}}=0, \quad a=x, y, z^{*}=0, h_{\mathrm{b}},\end{cases}
$$

hence

$$
\frac{\left.\partial^{4} \widetilde{U}_{a}^{0}\right|_{z^{*}}}{\partial \tilde{z}^{4}}-\left.\tilde{\kappa}^{4} \widetilde{U}_{a}^{0}\right|_{z^{*}}=0, \quad \text { with } \tilde{\kappa}=\left(\frac{4 \rho_{\mathrm{b}} \omega^{2}}{E_{\mathrm{b}} \hat{r}_{\mathrm{b}}^{2}}\right)^{1 / 4}
$$



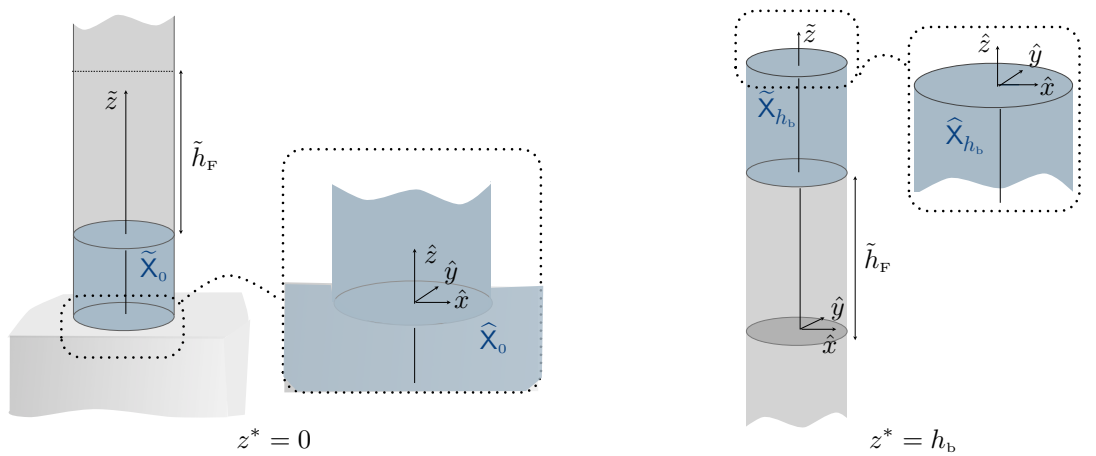

Figure 4: The intermediate regions and the microscopic regions near $z=z^{*}, z^{*}=0$ and $h_{\mathrm{b}}$. The intermediate regions $\widetilde{\mathrm{X}}_{0}$ and $\widetilde{\mathrm{X}}_{h_{\mathrm{b}}}$, correspond to the occurence of boundary layers as the flexural displacements loose their $\tilde{h}_{\mathrm{F}}$-periodicity (valid far from $z=z^{*}$. The microscopic regions, $\widehat{\mathrm{X}}_{0}$ and $\widehat{\mathrm{X}}_{h_{\mathrm{b}}}$, correspond to the classical boundary layers at the scale of $\ell$.

The periodic behavior is retrieved when $|\tilde{z}|$ goes to infinity, the solution in the intermediate region matching the $\tilde{z}$-periodic solution in the inner region of the beams

$$
\left(\widetilde{V}_{a}^{0}, \widetilde{M}_{a}^{0}, \widetilde{T}_{a}^{1}\right) \sim\left(U_{a}^{0}, M_{a}^{0}, T_{a}^{1}\right), \quad|\tilde{z}| \rightarrow \infty, a=x, y .
$$

Effective boundary conditions at $z=h_{b}$. OJO, j'en suis LA Near the top of the beams, each term of the expansion (30) satisfies the traction-free condition $\left.\widehat{\boldsymbol{\Sigma}}^{n}\right|_{h_{\mathrm{b}}} \mathbf{n}=\mathbf{0}$ on $\partial \hat{\mathrm{s}}_{\mathrm{b}}$. Next, the whole expansions match those of the intermediate problem, 24] (set in $\widetilde{\mathrm{X}}_{h_{\mathrm{b}}}$ ). We shall need the 
matching conditions for the stress only. Using $\tilde{z}=\eta \hat{z}$ in [24], re-expanding and identifying with [30, we get

$$
\left.\widetilde{\boldsymbol{\Sigma}}^{0}\right|_{h_{\mathrm{b}}}\left(\mathbf{x}^{\prime}, \hat{\mathbf{x}}^{\prime}, 0\right)=\left.\lim _{\hat{z} \rightarrow-\infty} \widehat{\boldsymbol{\Sigma}}^{0}\right|_{h_{\mathrm{b}}}\left(\mathbf{x}^{\prime}, \hat{\mathbf{x}}\right),\left.\quad \widetilde{\boldsymbol{\Sigma}}^{1}\right|_{h_{\mathrm{b}}}\left(\mathbf{x}^{\prime}, \hat{\mathbf{x}}^{\prime}, 0\right)=\lim _{\hat{z} \rightarrow-\infty}\left(\left.\widehat{\boldsymbol{\Sigma}}^{1}\right|_{h_{\mathrm{b}}}\left(\mathbf{x}^{\prime}, \hat{\mathbf{x}}\right)-\hat{z} \frac{\left.\partial \widetilde{\boldsymbol{\Sigma}}^{0}\right|_{h_{\mathrm{b}}}}{\partial \tilde{z}}\left(\mathbf{x}^{\prime}, \hat{\mathbf{x}}^{\prime}, 0\right)\right) .
$$

To derive the boundary conditions we only need the equations of equilibrium which read

$$
\left.(\mathrm{E})^{n} \quad \operatorname{div}_{\hat{\mathbf{x}}} \widehat{\boldsymbol{\Sigma}}^{n+2}\right|_{h_{\mathrm{b}}}+\left.\operatorname{div}_{\mathbf{x}^{\prime}} \widehat{\boldsymbol{\Sigma}}^{n}\right|_{h_{\mathrm{b}}}+\left.\rho_{\mathrm{b}} \omega^{2} \widehat{\mathbf{V}}^{n}\right|_{h_{\mathrm{b}}}=0 .
$$

124 (We implicitly set $\widehat{\boldsymbol{\Sigma}}^{m}=\mathbf{0}, \widehat{\mathbf{V}}^{m}=\mathbf{0}$ for $m<0$.)

At the dominant order, $n=0$ from 33 , $\left.\operatorname{div}_{\hat{\mathbf{x}}} \widehat{\boldsymbol{\Sigma}}^{0}\right|_{h_{\mathrm{b}}}=0$ that we integrate over $\widehat{\mathrm{X}}_{h_{\mathrm{b}}}$ to get $\mathbf{0}=$ $\left.\int_{\hat{X}_{h_{\mathrm{b}}}} \operatorname{div}_{\widehat{\mathbf{x}}} \widehat{\mathbf{\Sigma}}^{0}\right|_{h_{\mathrm{b}}} \mathrm{d} \hat{\mathbf{x}}=-\left.\lim _{\hat{z} \rightarrow-\infty} \int_{\hat{s}_{\mathrm{b}}} \widehat{\boldsymbol{\Sigma}}^{0}\right|_{h_{\mathrm{b}}} \mathbf{e}_{z} \mathrm{~d} \hat{\mathbf{x}}^{\prime}$. From the matching condition (32) at dominant order, we thus have $\left.\widetilde{T}_{a}^{0}\right|_{h_{\mathrm{b}}}\left(\mathbf{x}^{\prime}, 0\right)=\left.\int_{\hat{\mathrm{S}}_{\mathrm{b}}} \widetilde{\Sigma}_{a z}^{0}\right|_{h_{\mathrm{b}}}\left(\mathbf{x}^{\prime}, \hat{\mathbf{x}}^{\prime}, 0\right) \mathrm{d} \hat{\mathbf{x}}^{\prime}=0, a=x, y$, which is simply consistent with 27]. For the same reasons, we also get $\left.\widetilde{N}_{z z}^{0}\right|_{b}\left(\mathbf{x}^{\prime}, 0\right)=\int_{\hat{s}_{b}} \widetilde{\Sigma}_{z z}^{0} h_{b}\left(\mathbf{x}^{\prime}, \hat{\mathbf{x}}^{\prime}, 0\right) \mathrm{d} \hat{\mathbf{x}}^{\prime}=0$ from 27] and as $\widetilde{N}_{z z}^{0}$ does not depend on $\tilde{z}$ from 26 we eventually have that $\widetilde{N}_{z z}^{0} h_{\mathrm{b}}=N_{z z}^{0}\left(\mathbf{x}^{\prime}, h_{\mathrm{b}}\right)=0$. At the next order, we do the same (but we dont need $\left.N_{z z}^{1}\right)$; we use that $\frac{\left.\partial \widetilde{T}_{a}^{0}\right|_{h_{b}}}{\partial \tilde{z}^{\prime}}=0$ holds after derivation with respect to $\tilde{z}$ in 27$\}$ hence $\widetilde{T}_{a}^{1} l_{h_{\mathrm{b}}}\left(\mathbf{x}^{\prime}, 0\right)=\lim _{\hat{z} \rightarrow-\infty}\left(\int_{\hat{s}_{\mathrm{b}}} \widehat{\boldsymbol{\Sigma}}_{a z}^{1} \mid h_{\mathrm{b}} \mathrm{d} \hat{\mathbf{x}}^{\prime}-\hat{z} \int_{\hat{\mathrm{s}}_{\mathrm{b}}} \frac{\partial \widetilde{T}_{a}^{0} \mid l_{\mathrm{b}}}{\partial \tilde{z}}\left(\mathbf{x}^{\prime}, 0\right)\right)=0$ from the matching conditions [32]. At this stage we have obtained in the intermediate region at the top of the beams,

$$
\left.\widetilde{T}_{a}^{n}\right|_{h_{\mathrm{b}}}\left(\mathbf{x}^{\prime}, 0\right)=0, \quad a=x, y, n=0,1,\left.\quad \widetilde{N}_{z z}^{0}\right|_{h}\left(\mathbf{x}^{\prime}, \tilde{z}\right)=N_{z z}^{0}\left(\mathbf{x}^{\prime}, h_{\mathrm{b}}\right)=0 .
$$

To derive the boundary conditions on the bending moments, we come back to $\left.\operatorname{div}_{\hat{\mathbf{x}}} \widehat{\mathbf{\Sigma}}^{0}\right|_{h_{b}}=0$ that we multiply by $\boldsymbol{a}=-\hat{z} \mathbf{e}_{x}+\hat{x} \mathbf{e}_{z}$ and further integrate over $\widehat{X}_{h_{b}}$. By construction $\boldsymbol{\nabla} \boldsymbol{a}$ is antisymmetric, hence $\boldsymbol{\nabla} \boldsymbol{a} \widehat{\boldsymbol{\Sigma}}^{0}=0$. It follows that $0=\left.\int_{\partial \widehat{X}_{h_{\mathrm{b}}}} \boldsymbol{a} \cdot \widehat{\boldsymbol{\Sigma}}^{0}\right|_{h_{\mathrm{b}}} \mathbf{n} \mathrm{d} \hat{\mathbf{x}}=-\lim _{\hat{z} \rightarrow-\infty} \int_{\hat{\mathrm{s}}_{\mathrm{b}}}\left(\left.\hat{x} \widehat{\Sigma}_{z z}^{0}\right|_{h_{\mathrm{b}}}-\left.\hat{z} \widehat{\Sigma}_{x z}^{0}\right|_{h_{\mathrm{b}}}\right) \mathrm{d} \hat{\mathbf{x}}^{\prime}=$ $\left.\int_{\hat{\mathrm{s}}_{\mathrm{b}}} \hat{x} \widetilde{\Sigma}_{z z}^{0}\right|_{h_{\mathrm{b}}} \mathrm{d} \hat{\mathbf{x}}^{\prime}$ where we have accounted for $\left.\widetilde{T}_{x}^{0}\right|_{h_{\mathrm{b}}}\left(\mathbf{x}^{\prime}, 0\right)=\left.\lim _{\hat{z} \rightarrow-\infty} \int_{\hat{\mathrm{s}}_{\mathrm{b}}} \widehat{\Sigma}_{x z}^{0}\right|_{h_{\mathrm{b}}} \mathrm{d} \hat{\mathbf{x}}^{\prime}=0$ from (27) and (32), hence the boundary condition $\left.\widetilde{M}_{x}^{0}\right|_{h_{\mathrm{b}}}\left(\mathbf{x}^{\prime}, 0\right)=0$. The procedure can be repeated with $\boldsymbol{a}=-\hat{z} \mathbf{e}_{y}+\hat{y} \mathbf{e}_{z}$ resulting in $\widetilde{M}_{y}^{0} \mid h_{b}\left(\mathbf{x}^{\prime}, 0\right)=0$. We have thus obtained

$$
\left.\widetilde{M}_{a}^{0}\right|_{h_{\mathrm{b}}}\left(\mathbf{x}^{\prime}, 0\right)=0, \quad a=x, y .
$$

Effective transmission conditions at $z=0$. Near the interface between the beam and the substrate, we shall need the solution valid in the substrate far from $z=0$; this latter has variations associated to the typical wavelength only, hence the expansions are sought of the form

$$
\mathbf{u}=\sum_{n \geq 0} \eta^{n} \mathbf{u}^{n}(\mathbf{x}), \quad \boldsymbol{\sigma}=\sum_{n \geq 0} \eta^{n} \boldsymbol{\sigma}^{n}(\mathbf{x})
$$

We first determine the transmission conditions for the displacements. Given the mesoscopic and microscopic expansions (24) and [30, the matching conditions for the displacements between 
the soil, the microscopic region and the intermediate region read at the first orders

$$
\begin{cases}\left.\widetilde{\mathbf{V}}^{0}\right|_{0}\left(\mathbf{x}^{\prime}, \hat{\mathbf{x}}^{\prime}, 0\right)=\left.\lim _{\hat{z} \rightarrow+\infty} \widehat{\mathbf{V}}^{0}\right|_{0}\left(\mathbf{x}^{\prime}, \hat{\mathbf{x}}\right), & \mathbf{u}^{0}\left(\mathbf{x}^{\prime}, 0\right)=\left.\lim _{\hat{z} \rightarrow-\infty} \widehat{\mathbf{V}}^{0}\right|_{0}\left(\mathbf{x}^{\prime}, \hat{\mathbf{x}}\right), \\ \left.\widetilde{\mathbf{V}}^{1}\right|_{0}\left(\mathbf{x}^{\prime}, \hat{\mathbf{x}}^{\prime}, 0\right)=\lim _{\hat{z} \rightarrow+\infty}\left(\left.\widehat{\mathbf{V}}^{1}\right|_{0}\left(\mathbf{x}^{\prime}, \hat{\mathbf{x}}\right)-\hat{z} \frac{\left.\partial \widetilde{\mathbf{V}}^{0}\right|_{0}}{\partial \tilde{z}}\left(\mathbf{x}^{\prime}, \hat{\mathbf{x}}^{\prime}, 0\right)\right), & \mathbf{u}^{1}\left(\mathbf{x}^{\prime}, 0\right)=\left.\lim _{\hat{z} \rightarrow-\infty} \widehat{\mathbf{V}}^{1}\right|_{0}\left(\mathbf{x}^{\prime}, \hat{\mathbf{x}}\right) .\end{cases}
$$

In the microscopic region, the hierarchy of constitutive and equilibrium equations is given by

$$
\begin{cases}(\mathrm{C})^{n} & \left.\widehat{\boldsymbol{\Sigma}}^{n}\right|_{0}=\mathrm{A} \boldsymbol{\varepsilon}^{\hat{\mathbf{x}}}\left(\left.\widehat{\mathbf{V}}^{n+2}\right|_{0}\right)+\mathrm{A} \boldsymbol{\varepsilon}^{\mathbf{x}^{\prime}}\left(\left.\widehat{\mathbf{V}}^{n}\right|_{0}\right), \\ \left(\mathrm{C}^{\prime}\right)^{n} & \boldsymbol{\varepsilon}^{\hat{\mathbf{x}}}\left(\left.\widehat{\mathbf{V}}^{n+2}\right|_{0}\right)+\boldsymbol{\varepsilon}^{\mathbf{x}^{\prime}}\left(\left.\widehat{\mathbf{V}}^{n}\right|_{0}\right)=\left.\frac{1+v_{\mathrm{b}}}{E_{\mathrm{b}}} \widehat{\boldsymbol{\Sigma}}^{n}\right|_{0}-\frac{v_{\mathrm{b}}}{E_{\mathrm{b}}} \operatorname{tr}\left(\left.\widehat{\boldsymbol{\Sigma}}^{n}\right|_{0}\right) I .\end{cases}
$$

We have from $\left(\mathrm{C}^{\prime}\right)^{-2}$ and $\left(\mathrm{C}^{\prime}\right)^{-1}$ that $\left.\widehat{\mathbf{V}}^{n}\right|_{0}=\left.\widehat{\mathbf{\Omega}}^{n}\right|_{0}\left(\mathbf{x}^{\prime}\right) \times \hat{\mathbf{x}}+\left.\widehat{\mathbf{U}}^{n}\right|_{0}\left(\mathbf{x}^{\prime}\right), n=0,1$, are piecewise rigid body motions for $\hat{z}<0$ and $\hat{z}>0$. Invoking the periodic of $\left.\widehat{\mathbf{V}}^{n}\right|_{0}$ with respect to $\hat{\mathbf{x}}^{\prime}$ for $\hat{z}<0$ and the continuity of $\widehat{\mathbf{V}}^{n}$ at $\hat{z}=0$, these rigid body motions reduce to single translations $\left.\widehat{\mathbf{V}}^{n}\right|_{0}=\left.\widehat{\mathbf{U}}^{n}\right|_{0}\left(\mathbf{x}^{\prime}\right)$ independent of $\hat{\mathbf{x}}$. It follows from 377 that the beams are clamped to the substrate at the dominant order

$$
u_{a}^{0}\left(\mathbf{x}^{\prime}, 0\right)=\left.\widetilde{U}_{a}^{0}\right|_{0}\left(\mathbf{x}^{\prime}, 0\right), \quad \frac{\left.\partial \widetilde{U}_{a}^{0}\right|_{0}}{\partial \tilde{z}}\left(\mathbf{x}^{\prime}, 0\right)=0 .
$$

In particular, we have used that since $\left.\widehat{\mathbf{V}}^{1}\right|_{0}=\left.\widehat{\mathbf{U}}^{1}\right|_{0}\left(\mathbf{x}^{\prime}\right)$ does not contain any contribution linear in $\hat{z}$, then $\frac{\partial \widetilde{U}_{a}^{0}}{\partial \tilde{z}}$ has to cancel so that the matching condition (37) between $\left.\widehat{\mathbf{V}}^{1}\right|_{0}$ and $\left.\widetilde{\mathbf{V}}^{1}\right|_{0}\left(\mathbf{x}^{\prime}, \hat{\mathbf{x}}^{\prime}, 0\right)$ can be fulfilled .

We now establish transmission conditions on the stresses. The matching conditions for the stresses between the soil, the microscopic region and the intermediate region read

$$
\begin{cases}\left.\widetilde{\boldsymbol{\Sigma}}^{0}\right|_{0}\left(\mathbf{x}^{\prime}, \hat{\mathbf{x}}^{\prime}, 0\right)=\left.\lim _{\hat{z} \rightarrow+\infty} \widehat{\boldsymbol{\Sigma}}^{0}\right|_{0}\left(\mathbf{x}^{\prime}, \hat{\mathbf{x}}\right), & \sigma^{0}\left(\mathbf{x}^{\prime}, 0\right)=\left.\lim _{\hat{z} \rightarrow-\infty} \widehat{\boldsymbol{\Sigma}}^{0}\right|_{0}\left(\mathbf{x}^{\prime}, \hat{\mathbf{x}}\right), \\ \left.\widetilde{\boldsymbol{\Sigma}}^{1}\right|_{0}\left(\mathbf{x}^{\prime}, \hat{\mathbf{x}}^{\prime}, 0\right)=\lim _{\hat{z} \rightarrow+\infty}\left(\left.\widehat{\boldsymbol{\Sigma}}^{1}\right|_{0}\left(\mathbf{x}^{\prime}, \hat{\mathbf{x}}\right)-\hat{z} \frac{\left.\partial \widetilde{\boldsymbol{\Sigma}}^{0}\right|_{0}}{\partial \tilde{z}}\left(\mathbf{x}^{\prime}, \hat{\mathbf{x}}^{\prime}, 0\right)\right), & \sigma^{1}\left(\mathbf{x}^{\prime}, 0\right)=\left.\lim _{\hat{z} \rightarrow-\infty} \widehat{\boldsymbol{\Sigma}}^{1}\right|_{0}\left(\mathbf{x}^{\prime}, \hat{\mathbf{x}}\right) .\end{cases}
$$

By integration of $\left.\operatorname{div}_{\hat{\mathbf{x}}} \widehat{\boldsymbol{\Sigma}}^{0}\right|_{0}=\left.\operatorname{div}_{\widehat{\mathbf{x}}} \widehat{\boldsymbol{\Sigma}}^{1}\right|_{0}=0$ over $\widehat{\mathbf{X}}_{0}=\left\{\hat{\mathbf{x}}^{\prime} \in \hat{\mathrm{S}}_{\mathrm{b}}, \hat{z} \in(0,+\infty)\right\}$ and using 40 we get the following transmission conditions on the normal stress vector

$$
\sigma_{a z}^{0}\left(\mathbf{x}^{\prime}, 0\right)=\left.\hat{\mathcal{S}}^{-1} \widetilde{T}_{a}^{0}\right|_{0}\left(\mathbf{x}^{\prime}, 0\right)=0, \quad \sigma_{a z}^{1}\left(\mathbf{x}^{\prime}, 0\right)=\left.\hat{\mathcal{S}}^{-1} \widetilde{T}_{a}^{1}\right|_{0}\left(\mathbf{x}^{\prime}, 0\right), \quad a=x, y,
$$

${ }_{228}$ with $\hat{\mathcal{S}}=\hat{\ell}_{x} \times \hat{\ell}_{y}$ being the rescaled periodic section area and where we have accounted for $\frac{\left.\partial \widetilde{T}_{a}^{0}\right|_{0}}{\partial \tilde{z}}\left(\mathbf{x}^{\prime}, 0\right)=0, a=x, y$ from 27$)$.

\subsection{Construction of a unique problem}

We construct the problem at the dominant order for the displacement $U_{z}(\mathbf{x})=U_{z}^{0}(\mathbf{x})$ and $U_{a}(\mathbf{x})=\left(U_{a}\left(\mathbf{x}^{\prime}, \tilde{z}\right),\left.\widetilde{U}_{a}^{0}\right|_{0}\left(\mathbf{x}^{\prime}, \tilde{z}\right),\left.\widetilde{U}_{a}^{0}\right|_{h_{\mathrm{b}}}\left(\mathbf{x}^{\prime}, \tilde{z}\right)\right)$ accounting for $\tilde{z}=z / \eta$ and $r_{\mathrm{b}}=\eta^{2} \hat{r}_{\mathrm{b}}$. Gathering the longitudinal and flexural equations $(19),(23), 26),(28)$ in both the inner region of the beams 
and the intermediate region near the extremities, we get the final set of homogenized equations for $z \in\left(0, h_{\mathrm{b}}\right)$ on the

$$
\begin{cases}\frac{\partial^{4} U_{a}}{\partial z^{4}}-\kappa^{4} U_{a}=0, & \kappa^{4}=\frac{4 \rho_{\mathrm{b}} \omega^{2}}{E_{\mathrm{b}} r_{\mathrm{b}}^{2}}, \\ \frac{\partial^{2} U_{z}}{\partial z^{2}}+K^{2} U_{z}=0, & K^{2}=\frac{\rho_{\mathrm{b}}}{E_{\mathrm{b}}} \omega^{2} .\end{cases}
$$

Gathering the transmission conditions $(39)$ and $(41)$ at $z=0$ and free end boundary conditions (34) and (35) at $z=h_{\mathrm{b}}$ leads to the boundary conditions

$$
\left\{\begin{array}{lll}
\text { at } z=0, & U_{a}=u_{a}\left(\mathbf{x}^{\prime}, 0\right), \quad \frac{\partial U_{a}}{\partial z}=0, & \sigma_{a z}\left(\mathbf{x}^{\prime}, 0\right)=-\varphi E_{\mathrm{b}} \frac{r_{\mathrm{b}}^{2}}{4} \frac{\partial^{3} U_{a}}{\partial z^{3}}, \quad a=x, y, \\
& U_{z}=u_{z}\left(\mathbf{x}^{\prime}, 0\right), & \sigma_{z z}\left(\mathbf{x}^{\prime}, 0\right)=\varphi E_{\mathrm{b}} \frac{\partial U_{z}}{\partial z}, \\
\text { at } z=h_{\mathrm{b}}, & \frac{\partial^{2} U_{a}}{\partial z^{2}}=\frac{\partial^{3} U_{a}}{\partial z^{3}}=0, \quad \frac{\partial U_{z}}{\partial z}=0 . &
\end{array}\right.
$$

\section{Validation of the effective model}

In this section, we shall inspect the ability of our effective model to reproduce the actual scattering properties for an incident plane wave in a two-dimensional setting, with plates infinite along $y$ and incident waves in the $(x, z)$ plane (figure 5). In this case, we have

$$
\left\{\begin{array}{l}
\operatorname{div} \boldsymbol{\sigma}+\rho_{\mathrm{s}} \omega^{2} \mathbf{u}=\mathbf{0}, \quad \boldsymbol{\sigma}=2 \mu_{\mathrm{s}} \boldsymbol{\varepsilon}+\lambda_{\mathrm{s}} \operatorname{tr}(\boldsymbol{\varepsilon}) I, \quad \text { for } z \in(-\infty, 0), \\
\sigma_{x z}(x, 0)=\mu_{\mathrm{s}} k_{\mathrm{T}} f_{\mathrm{F}}\left(\omega, h_{\mathrm{b}}\right) u_{x}\left(\mathbf{x}^{\prime}, 0\right), \quad \sigma_{z z}(x, 0)=\mu_{\mathrm{s}} k_{\mathrm{T}} f_{\mathrm{L}}\left(\omega, h_{\mathrm{b}}\right) u_{z}(x, 0),
\end{array}\right.
$$

and

$$
\begin{cases}f_{\mathrm{F}}\left(\omega, h_{\mathrm{b}}\right)=\varphi \frac{\rho_{\mathrm{b}}}{\rho_{\mathrm{s}}} \frac{k_{\mathrm{T}}}{K} \frac{\operatorname{sh} \kappa h_{\mathrm{b}} \cos \kappa h_{\mathrm{b}}+\operatorname{ch} \kappa h_{\mathrm{b}} \sin \kappa h_{\mathrm{b}}}{1+\cos \kappa h_{\mathrm{b}} \operatorname{ch} \kappa h_{\mathrm{b}}}, & \kappa=\left(\frac{3 \rho_{\mathrm{b}} \omega^{2}}{E_{\mathrm{b}}^{*} r_{\mathrm{b}}^{2}}\right)^{1 / 4}, \\ f_{\mathrm{L}}\left(\omega, h_{\mathrm{b}}\right)=\varphi \frac{\rho_{\mathrm{b}}}{\rho_{\mathrm{s}}} \frac{k_{\mathrm{T}}}{K} \tan K h_{\mathrm{b}}, & K=\sqrt{\frac{\rho_{\mathrm{b}}}{E_{\mathrm{b}}^{*}}} \omega .\end{cases}
$$

with

$$
\varphi=\frac{2 r_{\mathrm{b}}}{\ell}, \quad E_{\mathrm{b}}^{*}=\frac{4 \mu_{\mathrm{b}}\left(\lambda_{\mathrm{b}}+\mu_{\mathrm{b}}\right)}{\lambda_{\mathrm{b}}+2 \mu_{\mathrm{b}}}=\frac{E_{\mathrm{b}}}{1-\nu_{\mathrm{b}}^{2}} .
$$

We use as reference case:

At the angular frequency $\omega=120$ rad. $\mathrm{s}^{-1}: \quad \ell=1 \mathrm{~m}, 2 r_{\mathrm{b}}=0.5 \mathrm{~m}$ and $h_{\mathrm{b}} \in(0,90) \mathrm{m}$,

Material properties: $\quad \rho_{\mathrm{b}}=500 \mathrm{~kg} \cdot \mathrm{m}^{-3}, E_{\mathrm{b}}=2 \mathrm{GPa}, v_{\mathrm{b}}=0.3$

$$
\rho_{\mathrm{s}}=1000 \mathrm{~kg} \cdot \mathrm{m}^{-3}, E_{\mathrm{s}}=2 \mathrm{GPa}, v_{\mathrm{s}}=0.2,
$$

resulting in $C=0.574$ and $\mathcal{K}=0.091$. 

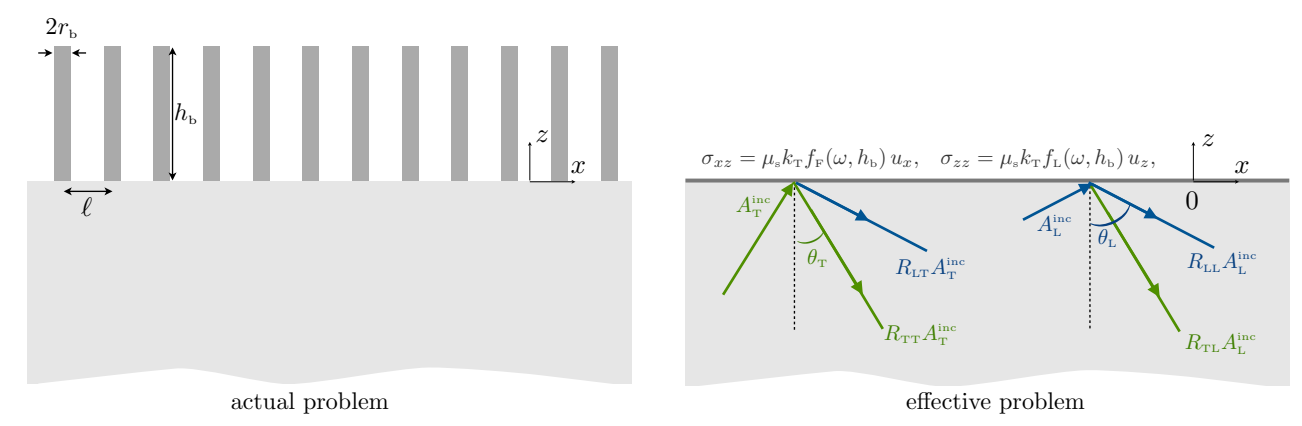

Figure 5: Scattering of an incident plane wave on an array of plates (two-dimensional settings); actual problem and effective problem with an interface ruled by 44 - 45 . The incident wave is characterized by $\left(A_{\mathrm{L}}, A_{\mathrm{T}}\right), 50$, and the solution reads 51 with four scattering coefficients 52 .

\subsection{Explicit solution of the effective problem}

To begin with, we stress that we dont have a one-to-one correspondance between frequency $\omega$ and height $h_{\mathrm{b}}$ as the wavelength associated to the flexural motions scales as $\sqrt{\omega} h_{\mathrm{b}}$ while that of the longitudinal motions scales as $\omega h_{\mathrm{b}}$. Next, we shall see that the effective conditions (44) involve two non-dimensional parameters and a non-dimensional frequency or a non-dimensional height.

\subsubsection{Result for given $h_{b}$ varying the frequency $\omega$}

From (44)-(45), we have

$$
\begin{cases}\text { with } C=\varphi \sqrt{\frac{\rho_{\mathrm{b}} E_{\mathrm{b}}^{*}}{\rho_{\mathrm{s}} \mu_{\mathrm{s}}}}, \quad \mathcal{S}=\frac{\sqrt{3} h_{\mathrm{b}}}{r_{\mathrm{b}}}, \quad \text { and } \quad \Omega=\left(\kappa h_{\mathrm{b}}\right)^{2}=\frac{\omega}{\omega_{0}}, \quad \omega_{0}=\frac{1}{\mathcal{S} h_{\mathrm{b}}} \sqrt{\frac{E_{\mathrm{b}}^{*}}{\rho_{\mathrm{b}}}} \\ f_{\mathrm{F}}(\Omega ; C, \mathcal{S})=C \frac{\sqrt{\Omega}}{\mathcal{S}} \frac{\operatorname{sh} \sqrt{\Omega} \cos \sqrt{\Omega}+\operatorname{ch} \sqrt{\Omega} \sin \sqrt{\Omega}}{1+\cos \sqrt{\Omega} \operatorname{ch} \sqrt{\Omega}}, \quad f_{\mathrm{L}}(\Omega ; C, \mathcal{S})=C \tan \left(\frac{\Omega}{\mathcal{S}}\right) .\end{cases}
$$

The parameter $C$ measures the effectiveness of the coupling between the plates and the substrate; higher coupling is obtained for heavier or stiffer plates. Next $\mathcal{S}$ is a measure of the slenderness of the plates; it does not affect the effectiveness of the longitudinal coupling between plates and substrate but large slendernesses weaken the flexural coupling. Eventually, the flexural resonances are given by constant $\sqrt{\Omega}_{\text {flex. res. }} \simeq \frac{\pi}{2}+n \pi, n$ integer, whatever the dimensions and material properties of the plates. In contrast, the positions of the longitudinal resonances with respect to the flexural ones depend on $\mathcal{S}$ through $\Omega_{\text {long. res. }}=\mathcal{S}\left(\frac{\pi}{2}+n \pi\right)$ (a larger slenderness produces a relative denser populations of flexural resonances, with $N \sim \sqrt{\mathcal{S}}$ flexural resonances between two longitudinal ones). 
4.1.2. Result for given $\omega$ and varying the height $h_{b}$

We adapt the preceding expressions as $\mathcal{S}$ and $\omega_{0}$ depend on $h_{\mathrm{b}}$ which now varies (at prescribed frequency) and we obtain

$$
\left\{\begin{array}{l}
\text { with } C=\varphi \sqrt{\frac{\rho_{\mathrm{b}} E_{\mathrm{b}}^{*}}{\rho_{\mathrm{s}} \mu_{\mathrm{s}}}}, \quad \mathcal{K}=\left(\frac{\left(K r_{\mathrm{b}}\right)^{2}}{3}\right)^{1 / 4}, \quad \text { and } \quad H=\left(\kappa h_{\mathrm{b}}\right), \\
f_{\mathrm{F}}(H ; C, \mathcal{K})=C \mathcal{K} \frac{\operatorname{sh} H \cos H+\operatorname{ch} H \sin H}{1+\cos H \operatorname{ch} H}, \quad f_{\mathrm{L}}(H ; C, \mathcal{K})=C \tan (\mathcal{K} H) .
\end{array}\right.
$$

The parameter $C$ is still involved as it determines the coupling due to the contrast in the material properties between the substrate and the plates. The new non-dimensional parameter $\mathcal{K}$ is a measure of the frequency regime through wavenumber $K=\omega \sqrt{\frac{\rho_{\mathrm{b}}}{E_{\mathrm{b}}^{*}}}$. Higher frequency longitudinal resonances take place for $\mathcal{K}$ large enough. The flexural resonances still take place at $H \sim \frac{\pi}{2}+n \pi$ and, at prescribed frequency, the density of flexural resonances between two longitudinal ones goes as $\frac{1}{\mathcal{K}}{ }^{2}$.

\subsubsection{Solution of the effective problem}

The scattering problem of an incident wave can be solved owing to the use of the Helmholtz decomposition for the elastic potentials $(\phi, \psi)$, with $\mathbf{u}=\boldsymbol{\nabla} \phi+\boldsymbol{\nabla} \times\left(\psi \mathbf{e}_{y}\right)$. The incident wave in the substrate is defined in terms of the incident potentials

$$
\left\{\begin{array}{l}
\phi^{\mathrm{inc}}(x, z)=A_{\mathrm{L}} e^{i \alpha_{\mathrm{L}} z} e^{i \beta x}, \quad \psi^{\mathrm{inc}}(x, z)=A_{\mathrm{T}} e^{i \alpha_{\mathrm{T}} z} e^{i \beta x}, \\
\text { with }\left(\alpha_{\mathrm{L}}, \beta\right)=k_{\mathrm{L}}\left(\cos \theta_{\mathrm{L}}, \sin \theta_{\mathrm{L}}\right), \quad\left(\alpha_{\mathrm{T}}, \beta\right)=k_{\mathrm{T}}\left(\cos \theta_{\mathrm{T}}, \sin \theta_{\mathrm{T}}\right),
\end{array}\right.
$$

and $k_{\mathrm{L}}=\sqrt{\frac{\rho_{\mathrm{s}}}{\lambda_{\mathrm{s}}+2 \mu_{\mathrm{s}}}} \omega$ and $k_{\mathrm{T}}=\sqrt{\frac{\rho_{\mathrm{s}}}{\mu_{\mathrm{s}}}} \omega$. The solution in the substrate reads

$$
\left\{\begin{array}{l}
\phi(x, z)=\phi^{\mathrm{inc}}(x, z)+\left(R_{\mathrm{LL}} A_{\mathrm{L}}+R_{\mathrm{LT}} A_{\mathrm{T}}\right) e^{-i \alpha_{\mathrm{L}} z} e^{i \beta x}, \\
\psi(x, z)=\psi^{\mathrm{inc}}(x, z)+\left(R_{\mathrm{TL}} A_{\mathrm{L}}+R_{\mathrm{TT}} A_{\mathrm{T}}\right) e^{-i \alpha_{\mathrm{T}} z} e^{i \beta x} .
\end{array}\right.
$$

The effective problem is a one dimensional problem and making use of (B.1) it can be solved explicitly, specifically we get

$$
\left\{\begin{array}{l}
R_{\mathrm{LL}}=\frac{1}{D}\left[\sin 2 \theta_{\mathrm{T}} \sin 2 \theta_{\mathrm{L}}-\xi^{2} \cos ^{2} 2 \theta_{\mathrm{T}}-i \xi\left(f_{\mathrm{L}} \cos \theta_{\mathrm{L}}-\xi f_{\mathrm{F}} \cos \theta_{\mathrm{T}}\right)-\xi f_{\mathrm{L}} f_{\mathrm{F}} \cos \left(\theta_{\mathrm{L}}+\theta_{\mathrm{T}}\right)\right] \\
R_{\mathrm{TT}}=\frac{1}{D}\left[\sin 2 \theta_{\mathrm{T}} \sin 2 \theta_{\mathrm{L}}-\xi^{2} \cos ^{2} 2 \theta_{\mathrm{T}}+i \xi\left(f_{\mathrm{L}} \cos \theta_{\mathrm{L}}-\xi f_{\mathrm{F}} \cos \theta_{\mathrm{T}}\right)-\xi f_{\mathrm{L}} f_{\mathrm{F}} \cos \left(\theta_{\mathrm{L}}+\theta_{\mathrm{T}}\right)\right] \\
R_{\mathrm{LT}}=-\frac{\xi^{2} \sin 2 \theta_{\mathrm{T}}}{D}\left(2 \cos 2 \theta_{\mathrm{T}}+f_{\mathrm{L}} f_{\mathrm{F}}\right), \quad R_{\mathrm{TL}}=\frac{\sin 2 \theta_{\mathrm{L}}}{D}\left(2 \cos 2 \theta_{\mathrm{T}}+f_{\mathrm{L}} f_{\mathrm{F}}\right), \\
\text { where } \quad D=\sin 2 \theta_{\mathrm{T}} \sin 2 \theta_{\mathrm{L}}+\xi^{2} \cos ^{2} 2 \theta_{\mathrm{T}}-i \xi\left(f_{\mathrm{L}} \cos \theta_{\mathrm{L}}+\xi f_{\mathrm{F}} \cos \theta_{\mathrm{T}}\right)-\xi f_{\mathrm{L}} f_{\mathrm{F}} \cos \left(\theta_{\mathrm{L}}-\theta_{\mathrm{T}}\right), \\
\text { with }\left(f_{\mathrm{L}}, f_{\mathrm{F}}\right) \text { in }(48) \text { or }(49), \text { and } \xi=\sqrt{\frac{\lambda_{\mathrm{s}}+2 \mu_{\mathrm{s}}}{\mu_{\mathrm{s}}}}
\end{array}\right.
$$

\footnotetext{
${ }^{2}$ The parameter $\mathcal{K}$ can be written $\mathcal{K}=\frac{K}{K}=\frac{h_{\mathrm{F}}}{h_{\mathrm{L}}}$; at prescribed frequency, it measures the relative wavelengths associated to flexural and longitudinal motions.
} 
We get the displacement fields in the substrate $z \in(-\infty, 0)$ with

$\left\{\begin{array}{l}u_{x}(x, z)=i k_{\mathrm{T}}\left[\sin \theta_{\mathrm{T}}\left(A_{\mathrm{L}} e^{i \alpha_{\mathrm{L}} z}+\left(R_{\mathrm{LL}} A_{\mathrm{L}}+R_{\mathrm{LT}} A_{\mathrm{T}}\right) e^{-i \alpha_{\mathrm{L}} z}\right)-\cos \theta_{\mathrm{T}}\left(A_{\mathrm{T}} e^{i \alpha_{\mathrm{T}} z}-\left(R_{\mathrm{TL}} A_{\mathrm{L}}+R_{\mathrm{TT}} A_{\mathrm{T}}\right) e^{-i \alpha_{\mathrm{T}} z}\right)\right], \\ u_{z}(x, z)=i k_{\mathrm{L}}\left[\cos \theta_{\mathrm{L}}\left(A_{\mathrm{L}} e^{i \alpha_{\mathrm{L}} z}-\left(R_{\mathrm{LL}} A_{\mathrm{L}}+R_{\mathrm{LT}} A_{\mathrm{T}}\right) e^{-i \alpha_{\mathrm{L}} z}\right)+\sin \theta_{\mathrm{L}}\left(A_{\mathrm{T}} e^{i \alpha_{\mathrm{T}} z}+\left(R_{\mathrm{TL}} A_{\mathrm{L}}+R_{\mathrm{TT}} A_{\mathrm{T}}\right) e^{-i \alpha_{\mathrm{T}} z}\right)\right],\end{array}\right.$

and in the region of the beams $z \in\left(0, h_{\mathrm{b}}\right)$, with

$$
\begin{gathered}
U_{x}(x, z)=u_{x}(x, 0) V_{\mathrm{L}}(z), \quad U_{z}(x, z)=u_{z}(x, 0) V_{\mathrm{F}}(z), \\
\left\{\begin{array}{c}
u_{x}(x, 0)=\frac{i k_{\mathrm{T}}}{D}\left[\sin 2 \theta_{\mathrm{L}}\left(2 \cos \theta_{\mathrm{T}}-i f_{\mathrm{L}}\right) A_{\mathrm{L}}-2 \xi \cos \theta_{\mathrm{T}}\left(\xi \cos 2 \theta_{\mathrm{T}}-i \cos \theta_{\mathrm{L}} f_{\mathrm{L}}\right) A_{\mathrm{T}}\right] e^{i \beta x}, \\
u_{z}(x, 0)=\frac{i \xi^{2} k_{\mathrm{L}}}{D}\left[2 \cos \theta_{\mathrm{L}}\left(\cos 2 \theta_{\mathrm{T}}-i \cos \theta_{\mathrm{T}} f_{\mathrm{F}}\right) A_{\mathrm{L}}+\sin 2 \theta_{\mathrm{T}}\left(2 \cos \theta_{\mathrm{L}}-i \xi f_{\mathrm{F}}\right) A_{\mathrm{T}}\right] e^{i \beta x} .
\end{array}\right.
\end{gathered}
$$

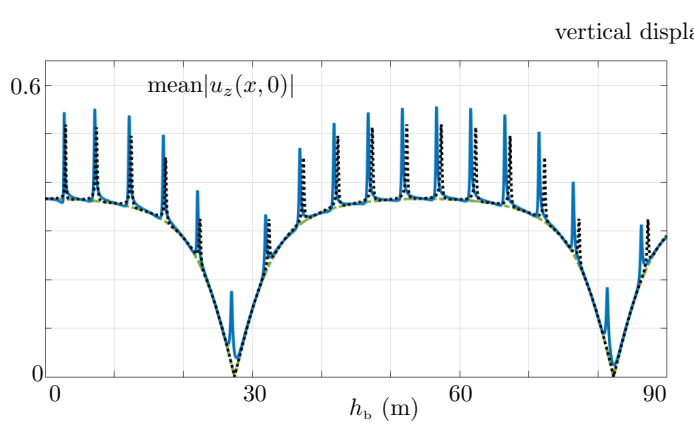

vertical displacement

To begin with, we come back to the representation of the figure 12 in [1] where we stressed the limit of the model to the occurence of the first longitudinal resonance and this limitation to low frequencies or small plates is now overcame 3 . We have computed numerically the actual displacements $u_{z}(x, 0)$ and $u_{z}\left(x, z_{0}\right)\left(z_{0}=-5 \mathrm{~m}\right)$ using the multimodal method presented in [15] in the reference case 477 . We used $\left(A_{\mathrm{L}}=\frac{1}{2 \beta}, A_{\mathrm{T}}=-\frac{1}{\alpha_{\mathrm{T}}}\right)$ resulting in an incident wave with unitary horizontal displacement at $z=0$. Results are reported in figure 6 together with the displacements given by the effective model $[53)$. For $h_{\mathrm{b}} \in(0,90) \mathrm{m}$, striking variations associated to the 17 flexural resonances are visible, being superimposed to an underlying smooth curve dictated by the first two longitudinal resonances at $h_{\mathrm{b}}=27.5 \mathrm{~m}$ and $82 \mathrm{~m}$. The overall agreement between

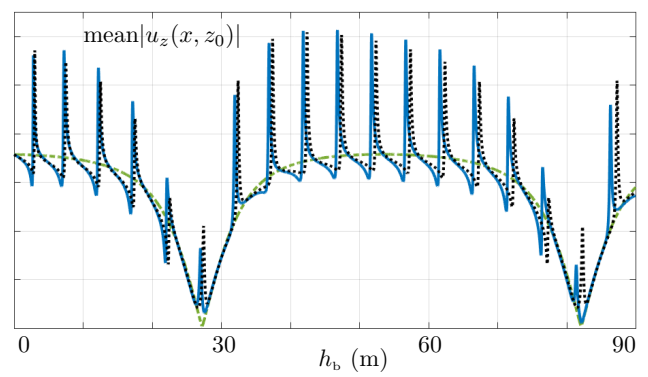

Figure 6: Variations of the mean vertical displacement $u_{z}$ at $z=0$ and $z=z_{0}=-5 \mathrm{~m}$ in the reference case, 47, for $\theta_{\mathrm{T}}=45^{\circ}$. Plain blue lines show $u_{z}$ computed numerically, dotted black lines the close forms in 53) and green dashed-dotted lines the same when neglecting the flexural motions of the plates $\left(f_{\mathrm{F}}=0\right.$ in 52 ).

the actual and the effective solutions is good although the actual resonances appear to be slightly shifted to frequencies lower than the predicted flexural resonances. We observe 11 resonances between the two first longitudinal resonances in agreement with a density $\frac{1}{\mathcal{K}}$, with $\mathcal{K} \simeq 0.092$

\footnotetext{
${ }^{3}$ At the occurence of the first longitudinal resonance, $f_{\mathrm{L}}$ diverges in 45 resulting in $u_{z}(x, 0)=0$ in 44 ; this was not captured in the model of [1] as $f_{\mathrm{L}}=\varphi \frac{\rho_{\mathrm{b}}}{\rho_{\mathrm{s}}} k_{\mathrm{T}} h_{\mathrm{b}}$ was obtained as an approximation of $f_{\mathrm{L}}$ in [44] for $K h_{\mathrm{b}} \ll 1$.
} 
from (48). Next, because of the low contrast in the material properties the coupling $C \simeq 0.574$ is weak, however the influence of both resonances is neat.

Eventually, a noticeable higher discrepancy is visible for $u_{z}(x, 0)$ than for $u_{z}\left(x, z_{0}=-5 \mathrm{~m}\right)$; in particular the actual displacement $u_{z}(x, 0)$ does not vanish at the longitudinal resonances for $h_{\mathrm{b}} \simeq 28 \mathrm{~m}$ and $82 \mathrm{~m}$ as predicted by the effective model. We have checked that removing the evanescent field from the actual solution, hence keeping only the propagating waves in the substrate, the agreement is better and in particular the condition $u_{z}(x, 0)=0$ is recovered. At the deeper position $z_{0}$, the evanescent field is negligible and the agreement is better.

We now move on to the influence of the parameter $(C, \mathcal{K})$ in $(48)$. We have computed the actual reflection coefficients varying the wave incidence and the height of the plates for 3 different radii. The spectra of $R_{\mathrm{TT}}$ and $R_{\mathrm{LL}}$ are reported in figures 7 and $8{ }^{4}$. The top panels show the actual
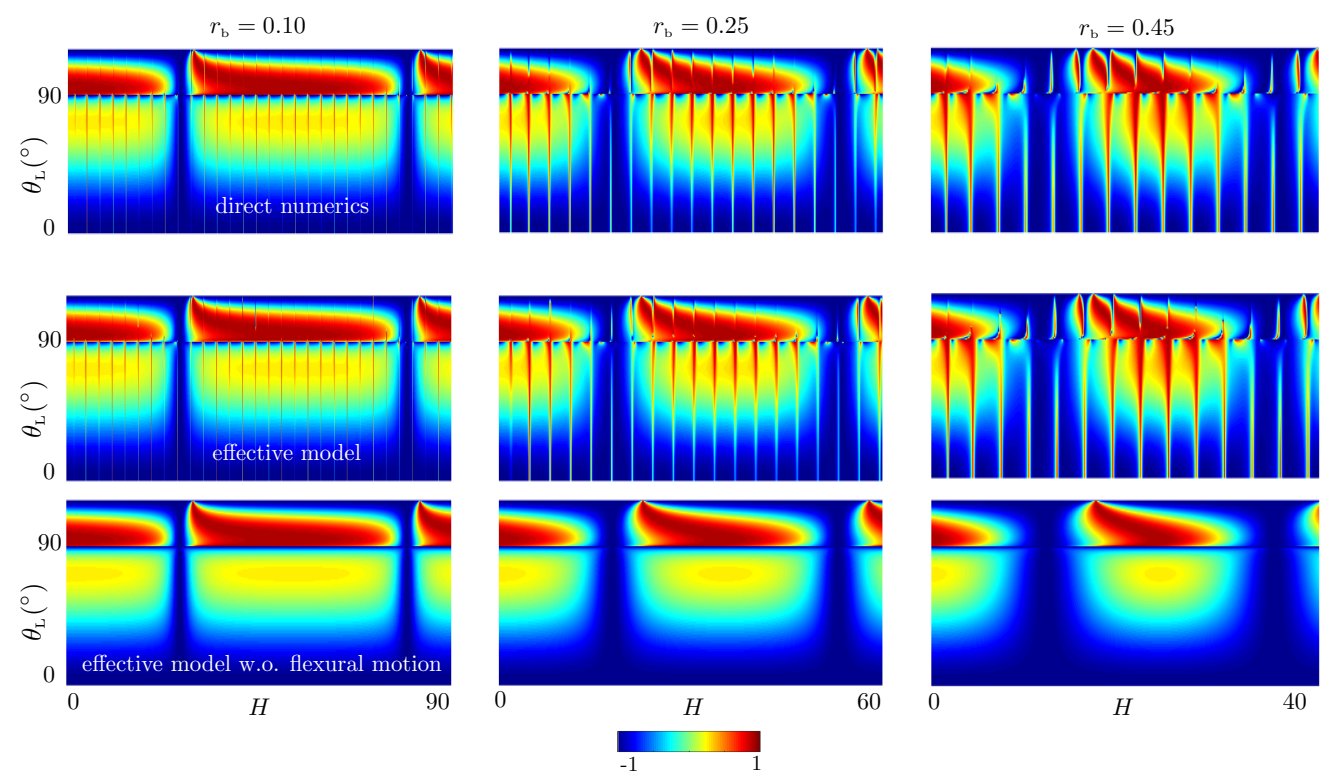

Figure 7: Real part of the reflection coefficient $R_{\mathrm{TT}}$ against $H=\kappa h_{\mathrm{b}}$ and the incidence angle $\theta_{\mathrm{L}} \in\left(0,90^{\circ}\right)$; the upper part of the spectra above $\theta_{\mathrm{L}}=90^{\circ}$ correspond to the range $\theta_{\mathrm{T}} \in\left(\theta_{c}, 90^{\circ}\right)$ with $\theta_{c} \simeq 37.8^{\circ}$ where longitudinal waves are evanescent (hence $\left|R_{\mathrm{TT}}\right|=1$ ).

spectra, together with those given by (52) (center panels). For comparison, the result when neglecting the flexural motions $\left(f_{\mathrm{L}}=0\right.$ in (52)) are shown in the bottom panels. With $h_{\mathrm{b}} \in(0,90)$ $\mathrm{m}$, the two first longitudinal resonances are visible at $\mathcal{K} H=\frac{\pi}{2}$ and $\frac{3 \pi}{2}$, with $\mathcal{K}=0.057,0.091$ and 0.122 ; accordingly, the density of flexural resonances between them decreases, with $N \simeq 17$, 11 and 8. The variations of $R_{\mathrm{TT}}$ and $R_{\mathrm{LL}}$ against $\theta_{\mathrm{L}}$ for the substrate on its own corresponds to $H=0$; for the thinest plates, $r_{\mathrm{b}}=0.10 \mathrm{~m}$, increasing $H$ does not affect much this scattering

\footnotetext{
${ }^{4}$ The spectrum of $R_{\mathrm{TT}}$ is reported against $\theta_{\mathrm{L}} \in\left(0,90^{\circ}\right)$ in its lower-part. As $\theta_{\mathrm{L}}=90^{\circ}, \theta_{\mathrm{T}}=\theta_{c}$ with $\theta_{c}=\operatorname{asin} \sqrt{\frac{\mu_{\mathrm{s}}}{\lambda_{\mathrm{s}}+2 \mu_{\mathrm{s}}}} \simeq$ $37.8^{\circ}$. Increasing further the incidence of the transverse wave above $\theta_{c}$, the longitudinal waves becomes evanescent and no mode conversion occurs (hence $\left|R_{\mathrm{TT}}\right|=1$ and the figure 7 shows the real part). Note that the expressions of the reflection coefficients in (52) remain valid, with $\sin \theta_{\mathrm{L}}=\frac{\beta}{k_{\mathrm{L}}}>1$, hence $\alpha_{\mathrm{L}}$ imaginary.
} 

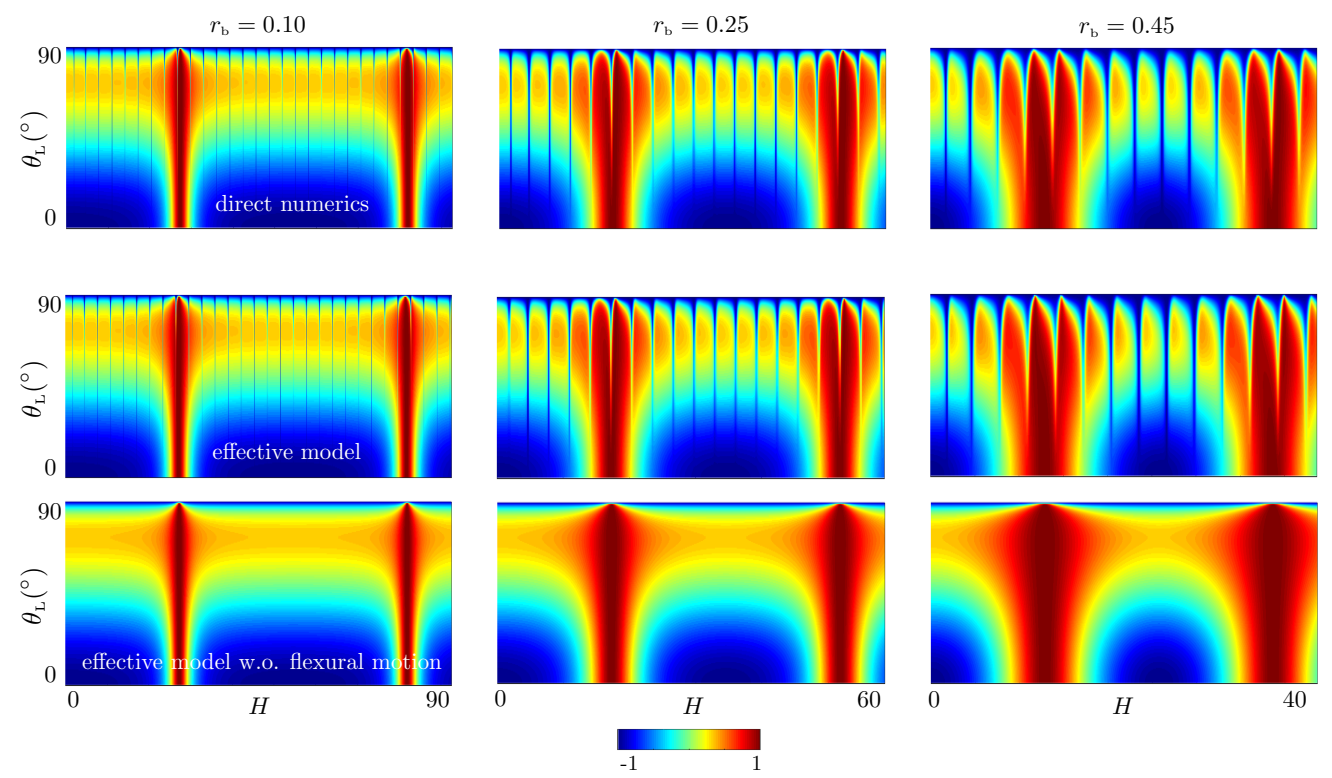

Figure 8: Real part of the reflection coefficient $R_{\mathrm{LL}}$; same representation as in figure 7

Another way to modify the coupling between the plates and the substrate is to increase the contrast in the material properties. We have increased both $\rho_{\mathrm{b}}$ and $E_{\mathrm{b}}$ by a factor 5 (from $C=$ 0.574 to 2.871 ), hence we have left identical the resonance frequencies. We report the results in figure 8 by means of the profiles of $\left(\left|R_{\mathrm{TT}}\right|,\left|R_{\mathrm{LT}}\right|\right)$ against $H$ for $\theta_{\mathrm{T}}=25.6^{\circ}\left(\theta_{\mathrm{L}}=45^{\circ}\right)$. In the reference case with moderate contrast, the scars at the flexural resonances already produce a significant shift from the smooth underlying curve when accounting for $f_{\mathrm{L}}$ only (dashed-dotted green line). In particular, it is noticeable that the reflexion coefficients do not follow the simple prediction $\left|R_{\mathrm{TT}}\right|=1,\left|R_{\mathrm{LT}}\right|=0$ obtained when neglecting the flexural motions (dashed-dotted green lines). For $\left|f_{\mathrm{L}}\right| \rightarrow+\infty$, (52) simplify to

$$
R_{\mathrm{TT}}=\frac{\cos \theta_{\mathrm{L}}-i f_{\mathrm{F}} \cos \left(\theta_{\mathrm{L}}+\theta_{\mathrm{T}}\right)}{\cos \theta_{\mathrm{L}}-i f_{\mathrm{F}} \cos \left(\theta_{\mathrm{L}}-\theta_{\mathrm{T}}\right)}, \quad R_{\mathrm{LT}}=\frac{-2 i f_{\mathrm{F}} \sin \theta_{\mathrm{L}} \cos \theta_{\mathrm{T}}}{\cos \theta_{\mathrm{L}}-i f_{\mathrm{F}} \cos \left(\theta_{\mathrm{L}}-\theta_{\mathrm{T}}\right)},
$$

hence their values depend on the relative position of the longitudinal resonance with the surrounding flexural ones. Eventually, for strong contrast, the variations due longitudinal motions only do not share much with the actual ones, as the influence of the flexural resonances are not reduced anymore to local scars. It is worth noting that the discrepancy between our model (dotted black lines) and that neglecting flexural motions (dashed-dotted green lines) is more important for the reported absolute values than for the real parts in figures 8 and 9 . This is due to the fact that the imaginary parts of the reflexion coefficients have more pronounced variations around the resonances (they cancel for the substrate on its own, (52)). 

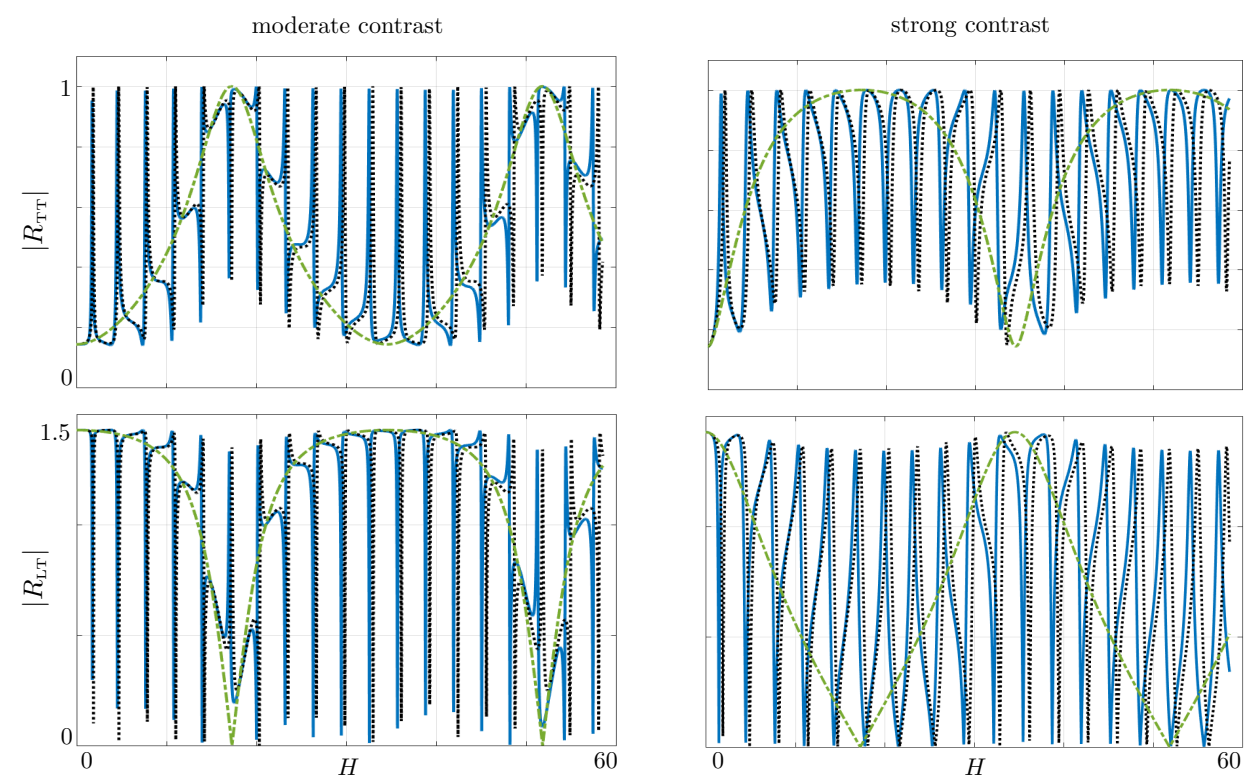

Figure 9: Variations of $\left(\left|R_{\mathrm{TT}}\right|,\left|R_{\mathrm{LT}}\right|\right)$ against $H$ for a moderate contrast, 47, and for a strong contrast (see main text) Plain blue lines shows the actual coefficients, dotted black lines those given by [52) and the dashed-dotted green lines the same when omitting the flexural motion.

We end this section with a slightly different representation by inspecting the conservation of the energy fluxes. Conservation of the fluxes is measured in terms of the conservation of the fluxes of the Poynting vector $\Pi=\int_{z<0} \mathfrak{I}\left(\sigma_{x z} u_{x}+\sigma_{z z} u_{z}\right) \mathrm{d} x$ where $\mathfrak{I}$ means imaginary parts which, from (53), reads

$$
\mathfrak{R}\left(\alpha_{\mathrm{T}}\left|R_{\mathrm{TT}}\right|^{2}+\alpha_{\mathrm{L}}\left|R_{\mathrm{LT}}\right|^{2}\right) A_{\mathrm{T}}^{2}=\mathfrak{R}\left(\alpha_{\mathrm{T}} A_{\mathrm{T}}^{2}\right), \quad \mathfrak{R}\left(\alpha_{\mathrm{L}}\left|R_{\mathrm{LL}}\right|^{2}+\alpha_{\mathrm{T}}\left|R_{\mathrm{TL}}\right|^{2}\right) A_{\mathrm{L}}^{2}=\mathfrak{R}\left(\alpha_{\mathrm{L}} A_{\mathrm{L}}^{2}\right),
$$

(where $\mathfrak{R}$ means real part) and the results holds by summing the two equations for an incident flux $\mathfrak{R}\left(\alpha_{\mathrm{T}} A_{\mathrm{T}}^{2}+\alpha_{\mathrm{L}} A_{\mathrm{L}}^{2}\right)$. For an incident transverse wave, we have computed the normalized energy fluxes

$$
\Phi_{\mathrm{TT}}=\left|R_{\mathrm{TT}}\right|^{2}, \quad \Phi_{\mathrm{LT}}=\mathfrak{R}\left(\frac{\alpha_{\mathrm{L}}}{\alpha_{\mathrm{T}}}\right)\left|R_{\mathrm{LT}}\right|^{2},
$$

and the conservation of the fluxes is ensured by $\Phi_{\mathrm{TT}}+\Phi_{\mathrm{LT}}=1$. The figure 10 shows the variations of $\left(\Phi_{\mathrm{TT}}, \Phi_{\mathrm{LT}}\right)$ against $H$ in the reference case for an incidence $\theta_{\mathrm{L}}=30^{\circ}, 60^{\circ}$ and $88^{\circ}$ (corresponding to $\theta_{\mathrm{T}}=17.8^{\circ}, 32.0^{\circ}$ and $37.7^{\circ}$ ). For the substrate on its own, the conversion from transverse to longitudinal wave is important, $\Phi_{\mathrm{TT}} \sim 0.1, \Phi_{\mathrm{LT}} \sim 0.9$ depending on the incidence. The presence of the array drastically modifies the exchanges of energy between the two polarizations. In general, the sharpest variations take place in the vicinity of the flexural resonances where the conversion is forbidden. However, as $H$ or $\theta_{\mathrm{T}}$ increases, less expected behaviors are observed, as the almost complete conversion $\Phi_{\text {Іт }} \simeq 1$ preceding or following the zero conversion at flexural resonances (see $\theta_{\mathrm{T}}=32.0^{\circ}, H>20$ ). 

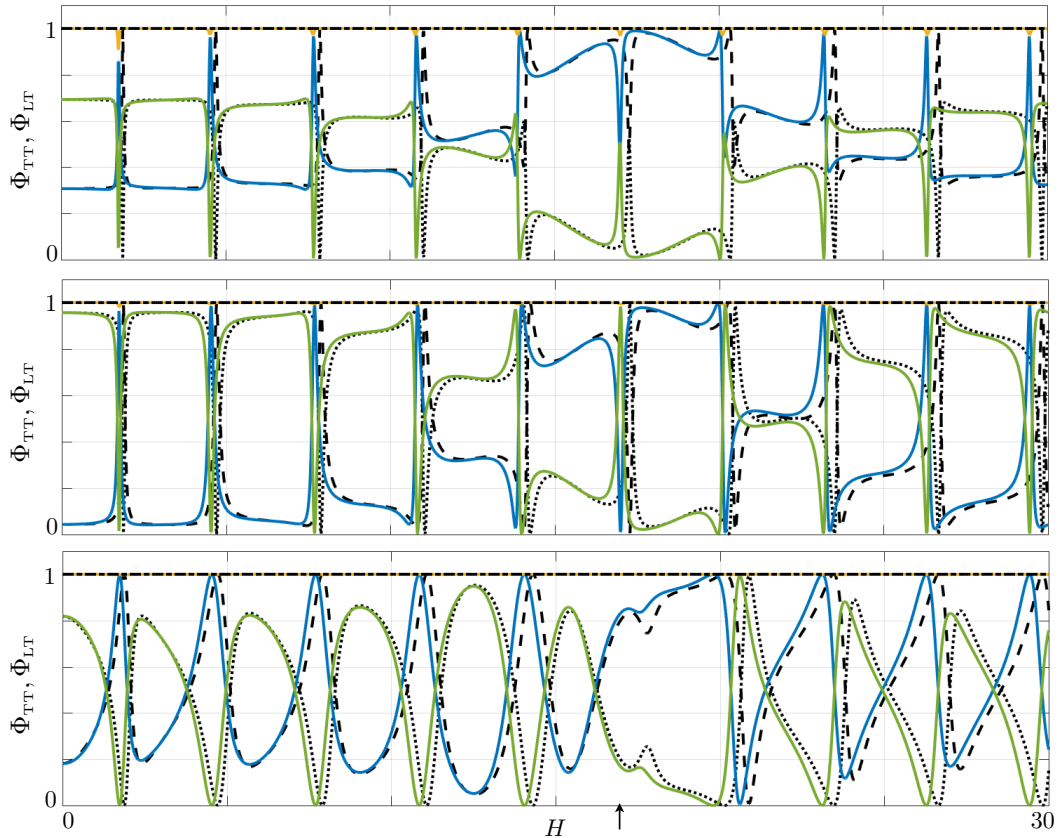

Figure 10: Variation of the energy fluxes $\Phi_{\mathrm{TT}}$ (blue lines) and $\Phi_{\mathrm{LT}}$ (green lines) against $H$ in the reference case, 47], for $\theta_{\mathrm{T}}=17.8^{\circ}$ (top panel), $\theta_{\mathrm{T}}=32.0^{\circ}$ (center panel) and $\theta_{\mathrm{T}}=37.7^{\circ}$ (bottom panel). The dashed and dotted black lines show $\Phi_{\mathrm{TT}}$ and $\Phi_{\mathrm{LT}}$ in the effective model with (52) in (56). The yellow and dashed-dotted black lines show $\left(\Phi_{\mathrm{TT}}+\Phi_{\mathrm{LT}}\right)$ in the numerics and in our model. The arrow indicates the position of the longitudinal resonance.

\section{Appendix A. Proofs of the properties in 16}

Throughout the appendix, we shall use that for a symmetric tensor $\tau_{i j}$ defined for $\hat{\mathbf{x}}^{\prime} \in \hat{\mathrm{s}}_{\mathrm{b}}$, and for a vector $a_{i}$ with antisymmetric tensor $\partial_{j} a_{i}$, we have

$$
\int_{\hat{\mathrm{s}}_{\mathrm{b}}} a_{i} \partial_{j} \tau_{i j} \mathrm{~d} \hat{\mathbf{x}}^{\prime}=\int_{\partial \hat{\mathrm{s}}_{\mathrm{b}}} a_{i} \tau_{i j} n_{j} \mathrm{~d} \hat{\mathbf{x}}^{\prime}-\int_{\partial \hat{\mathbf{s}}_{\mathrm{b}}} \partial_{j} a_{i} \tau_{i j} \mathrm{~d} \hat{\mathbf{x}}^{\prime}=\int_{\partial \hat{\mathbf{s}}_{\mathrm{b}}} a_{i} \tau_{i j} n_{j} \mathrm{~d} \hat{\mathbf{x}}^{\prime},
$$

since $\partial_{j} a_{i} \tau_{i j}=0$.

\section{Appendix A.1. Determination of $\left(\mathbf{V}^{0}, \mathbf{V}^{1}\right)$ and of $\left(\mathbf{\Sigma}^{0}, \mathbf{V}^{2}\right)$}

As previously said in $\$ 3.1 .3$, the dependance of $\mathbf{V}^{0}$ and $\mathbf{V}^{1}$ on $\hat{\mathbf{x}}^{\prime}=(\hat{x}, \hat{y})$ is obtained starting from $\left(C^{\prime}\right)^{-2}$ and $\left(C^{\prime}\right)^{-1}$ in 140 which tell us that there exists $\left(\mathbf{U}^{0}(\mathbf{x}, \tilde{z}), \Omega^{0}(\mathbf{x}, \tilde{z})\right)$ and $\left(\mathbf{U}^{1}(\mathbf{x}, \tilde{z}), \Omega^{1}(\mathbf{x}, \tilde{z})\right)$ such that

$$
\begin{cases}V_{x}^{0}=U_{x}^{0}(\mathbf{x}, \tilde{z})+\Omega^{0}(\mathbf{x}, \tilde{z}) \hat{y}, & V_{y}^{0}=U_{y}^{0}(\mathbf{x}, \tilde{z})-\Omega^{0}(\mathbf{x}, \tilde{z}) \hat{x}, \\ V_{x}^{1}=U_{x}^{1}(\mathbf{x}, \tilde{z})+\Omega^{1}(\mathbf{x}, \tilde{z}) \hat{y}, & V_{y}^{1}=U_{y}^{1}(\mathbf{x}, \tilde{z})-\Omega^{1}(\mathbf{x}, \tilde{z}) \hat{x} .\end{cases}
$$

Next, as $\frac{\partial}{\partial \hat{x}} V_{z}^{1}=-\frac{\partial}{\partial \tilde{z}} V_{x}^{0}, \frac{\partial}{\partial \hat{y}} V_{z}^{1}=-\frac{\partial}{\partial \tilde{z}} V_{y}^{0}$ from $\left(C^{\prime}\right)^{-1}$, we have $\frac{\partial^{2}}{\partial \hat{x} \partial \hat{y}} V_{z}^{1}= \pm \frac{\partial}{\partial \tilde{z}} \Omega^{0}=0$, hence

$$
\frac{\partial \Omega^{0}}{\partial \tilde{z}}=0, \quad V_{z}^{1}=U_{z}^{1}(\mathbf{x}, \tilde{z})-\frac{\partial}{\partial \tilde{z}}\left(U_{x}^{0} \hat{x}+U_{y}^{0} \hat{y}\right) .
$$


The dependance of $\mathbf{V}^{2}$ with respect to $\hat{\mathbf{x}}^{\prime}$ is more intricate as it requires to solve the following problem set in the section $\hat{\mathbf{x}}^{\prime} \in \hat{\mathrm{s}}_{\mathrm{b}}$ with $\left(\boldsymbol{\Sigma}^{0}, \mathbf{V}^{2}\right)$ as unknowns :

$$
\left\{\begin{array}{l}
\operatorname{div}_{\hat{\mathbf{x}}^{\prime}} \boldsymbol{\Sigma}^{0}=\mathbf{0} \quad \text { in } \hat{\mathrm{S}}_{\mathrm{b}}, \quad \boldsymbol{\Sigma}^{0} \mathbf{n}=\mathbf{0} \quad \text { on } \partial \hat{\mathbf{S}}_{\mathrm{b}}, \\
\varepsilon^{\hat{\mathbf{x}}^{\prime}}\left(\mathbf{V}^{2}\right)+\varepsilon^{\tilde{z}}\left(\mathbf{V}^{1}\right)+\varepsilon^{\mathbf{x}}\left(\mathbf{V}^{0}\right)=\frac{1+v_{\mathrm{b}}}{E_{\mathrm{b}}} \boldsymbol{\Sigma}^{0}-\frac{v_{\mathrm{b}}}{E_{\mathrm{b}}} \operatorname{tr}\left(\boldsymbol{\Sigma}^{0}\right) I,
\end{array}\right.
$$

with the input $\mathbf{V}^{0}$ and $\mathbf{V}^{1}$ given by (A.2). For a circular section, this problem can be solved explicitly. Specifically there exists $\left(\mathbf{U}^{2}(\mathbf{x}, \tilde{z}), \Omega^{2}(\mathbf{x}, \tilde{z})\right)$ such that the displacement $\mathbf{V}^{2}$ is given by

$$
\left\{\begin{aligned}
V_{x}^{2}= & U_{x}^{2}(\mathbf{x}, \tilde{z})+\Omega^{2}(\mathbf{x}, \tilde{z}) \hat{y}-\frac{\partial U_{x}^{0}}{\partial x} \hat{x}-\left(\frac{\partial U_{y}^{0}}{\partial x}+\frac{\partial U_{x}^{0}}{\partial y}\right) \frac{\hat{y}}{2}-\left(\frac{\partial \Omega^{0}}{\partial x} \hat{x}+\frac{\partial \Omega^{0}}{\partial y} \hat{y}\right) \hat{y} \\
& -v_{\mathrm{b}}\left(\frac{\partial U_{z}^{1}}{\partial \tilde{z}}+\frac{\partial U_{z}^{0}}{\partial z}\right) \hat{x}+v_{\mathrm{b}} \frac{\partial^{2}}{\partial \tilde{z}^{2}}\left(U_{x}^{0} \frac{\hat{x}^{2}-\hat{y}^{2}}{2}+U_{y}^{0} \hat{x} \hat{y}\right) \\
V_{y}^{2}= & U_{y}^{2}(\mathbf{x}, \tilde{z})-\Omega^{2}(\mathbf{x}, \tilde{z}) \hat{x}-\frac{\partial U_{y}^{0}}{\partial y} \hat{y}-\left(\frac{\partial U_{y}^{0}}{\partial x}+\frac{\partial U_{x}^{0}}{\partial y}\right) \frac{\hat{x}}{2}+\left(\frac{\partial \Omega^{0}}{\partial x} \hat{x}+\frac{\partial \Omega^{0}}{\partial y} \hat{y}\right) \hat{x} \\
& -v_{\mathrm{b}}\left(\frac{\partial U_{z}^{1}}{\partial \tilde{z}}+\frac{\partial U_{z}^{0}}{\partial z}\right) \hat{y}+v_{\mathrm{b}} \frac{\partial^{2}}{\partial \tilde{z}^{2}}\left(U_{x}^{0} \hat{x} \hat{y}-U_{y}^{0} \frac{\hat{x}^{2}-\hat{y}^{2}}{2}\right), \\
V_{z}^{2}= & U_{z}^{2}(\mathbf{x}, \tilde{z})-\left(\frac{\partial U_{x}^{1}}{\partial \tilde{z}}+\frac{\partial U_{z}^{0}}{\partial x}+\frac{\partial U_{x}^{0}}{\partial z}\right) \hat{x}-\left(\frac{\partial U_{y}^{1}}{\partial \tilde{z}}+\frac{\partial U_{z}^{0}}{\partial y}+\frac{\partial U_{y}^{0}}{\partial z}\right) \hat{y}
\end{aligned}\right.
$$

and the corresponding stress tensor $\boldsymbol{\Sigma}^{0}$ by

$$
\left\{\begin{array}{l}
\Sigma_{x z}^{0}=\mu_{\mathrm{b}}\left(\frac{\partial \Omega^{0}}{\partial z}+\frac{\partial \Omega^{1}}{\partial \tilde{z}}\right) \hat{y}, \quad \Sigma_{y z}^{0}=-\mu_{\mathrm{b}}\left(\frac{\partial \Omega^{0}}{\partial z}+\frac{\partial \Omega^{1}}{\partial \tilde{z}}\right) \hat{x} \\
\Sigma_{z z}^{0}=E_{\mathrm{b}}\left(\frac{\partial U_{z}^{1}}{\partial \tilde{z}}+\frac{\partial U_{z}^{0}}{\partial z}-\frac{\partial^{2}}{\partial \tilde{z}^{2}}\left(U_{x}^{0} \hat{x}+U_{y}^{0} \hat{y}\right)\right), \quad \Sigma_{x x}^{0}=\Sigma_{x y}^{0}=\Sigma_{y y}^{0}=0 .
\end{array}\right.
$$

Appendix A.2. Proof of $P_{1}: \frac{\partial U_{z}^{1}}{\partial z}=0$ in $(16)$

We use $\left(\mathrm{E}_{z}\right)^{-1}$ in $(13)$, specifically

$$
\frac{\partial}{\partial \hat{x}} \Sigma_{x z}^{1}+\frac{\partial}{\partial \hat{y}} \Sigma_{y z}^{1}+\frac{\partial}{\partial z} \Sigma_{z z}^{0}=0
$$

that we integrate over $\hat{\mathbf{x}}^{\prime} \in \hat{\mathrm{s}}_{\mathrm{b}}$. Accounting for the boundary condition $\Sigma_{x z}^{1} n_{x}+\Sigma_{y z}^{1} n_{y}=0$ on $\partial \hat{\mathrm{s}}_{\mathrm{b}}$, we obtain that $\frac{\partial}{\partial \tilde{z}} \int_{\hat{s}_{\mathrm{b}}} \Sigma_{z z}^{0} \mathrm{~d} \hat{\mathbf{x}}^{\prime}=0$. Next, with the form of $\Sigma_{z z}^{0}$ given in A.5, and accounting for the facts that (i) $U_{z}^{0}$ in A.2 does not depend on $\tilde{z}$ and (ii) $\int_{\hat{S}_{\mathrm{b}}} \hat{x} \mathrm{~d} \hat{\mathbf{x}}^{\prime}=\int_{\hat{\mathrm{s}}_{\mathrm{b}}} \hat{y} \mathrm{~d} \hat{\mathbf{x}}^{\prime}=0$, we obtain that $\frac{\partial^{2} U_{z}^{1}}{\partial \tilde{z}^{2}}=0$. The field $U_{z}^{1}$ could be a linear function of $\tilde{z}$ but this is prevented by its $\tilde{z}$-periodicity hence

$$
\frac{\partial U_{z}^{1}}{\partial \tilde{z}}=0
$$


214 Appendix A.3. Proof of $P_{2}: \frac{\partial \Omega^{1}}{\partial \tilde{z}}=0$ in $(16)$

We start by introducing the torsion force $\mathcal{T}^{0}$

$$
\mathcal{T}^{0}=\int_{\hat{\mathrm{s}}_{\mathrm{b}}}\left(\hat{y} \Sigma_{x z}^{0}-\hat{x} \Sigma_{y z}^{0}\right) \mathrm{d} \hat{\mathbf{x}}^{\prime}
$$

Using the forms of $\Sigma_{x z}^{0}$ and $\Sigma_{y z}^{0}$ in A.5 we get that

$$
\mathcal{T}^{0}(\mathbf{x}, \tilde{z})=\mu_{\mathrm{b}} J_{\mathrm{b}}\left(\frac{\partial \Omega^{0}}{\partial z}(\mathbf{x})+\frac{\partial \Omega^{1}}{\partial \tilde{z}}(\mathbf{x}, \tilde{z})\right), \quad \text { with } J_{\mathrm{b}}=\int_{\hat{\mathrm{s}}_{\mathrm{b}}}\left(\hat{x}^{2}+\hat{y}^{2}\right) \mathrm{d} \hat{\mathbf{x}}^{\prime}=\frac{\pi}{2} \hat{r}_{\mathrm{b}}^{4} .
$$

( $J_{\mathrm{b}}$ is the torsion constant for a circular cross-section.) Now, we use $\operatorname{div}_{\hat{\mathbf{x}}^{\prime}} \boldsymbol{\Sigma}^{1}+\operatorname{div}_{\tilde{z}} \boldsymbol{\Sigma}^{0}=\mathbf{0}$, from $\left(\mathrm{E}_{a}\right)^{-1}$ and $\left(\mathrm{E}_{z}\right)^{-1}$ in $(13)$, that we integrate over $\hat{\mathrm{s}}_{\mathrm{b}}$ after multiplication by $\boldsymbol{a}=\hat{y} \mathbf{e}_{x}-\hat{x} \mathbf{e}_{y}$; we get

$$
0=\int_{\hat{\mathrm{s}}_{\mathrm{b}}} \boldsymbol{a} \cdot \operatorname{div}_{\hat{\mathbf{x}}^{\prime}} \boldsymbol{\Sigma}^{1} \mathrm{~d} \hat{\mathbf{x}}^{\prime}+\int_{\hat{\mathrm{s}}_{\mathrm{b}}} \boldsymbol{a} \cdot \operatorname{div}_{\tilde{z}} \boldsymbol{\Sigma}^{0} \mathrm{~d} \hat{\mathbf{x}}^{\prime}=\int_{\partial \hat{\mathrm{s}}_{\mathrm{b}}} \boldsymbol{a} \cdot\left(\boldsymbol{\Sigma}^{1} \mathbf{n}\right) \mathrm{d} \hat{\mathbf{x}}^{\prime}+\frac{\partial}{\partial \tilde{z}} \int_{\hat{\mathrm{s}}_{\mathrm{b}}} \boldsymbol{a} \cdot\left(\boldsymbol{\Sigma}^{0} \mathbf{e}_{z}\right) \mathrm{d} \hat{\mathbf{x}}^{\prime}
$$

In A.9, we have applied A.1 to the first integral involving $\Sigma^{1}$. Indeed, it suffices to notice that $\operatorname{div}_{\hat{\mathbf{x}}^{\prime}} \boldsymbol{\Sigma}^{1}=\left.\operatorname{div}_{\hat{\mathbf{x}}^{\prime}} \boldsymbol{\Sigma}^{1}\right|_{\hat{\mathbf{x}}^{\prime}}$ where $\left.\boldsymbol{\Sigma}^{1}\right|_{\hat{\mathbf{x}}^{\prime}}$ is the $2 \times 2$ symmetric restriction of $\boldsymbol{\Sigma}^{1}$ to the indices $(x, y)$ and that $\boldsymbol{\nabla}_{\hat{\mathbf{x}}^{\prime}} \boldsymbol{a}$ is antisymmetric. Since $\left.\boldsymbol{\Sigma}^{1}\right|_{\hat{\mathbf{x}}^{\prime}} \mathbf{n}=\boldsymbol{\Sigma}^{1} \mathbf{n}=\mathbf{0}$ over $\partial \hat{\mathbf{s}}_{\mathrm{b}}$, we deduce that $\int_{\partial \hat{\mathrm{s}}_{\mathrm{b}}} \boldsymbol{a} \cdot\left(\boldsymbol{\Sigma}^{1} \mathbf{n}\right) \mathrm{d} \hat{\mathbf{x}}^{\prime}=0$. Now, recalling the definition A.7 of $\mathcal{T}^{0}$ as well as A.8, we find

$$
0=\frac{\partial}{\partial \tilde{z}} \int_{\hat{\mathrm{s}}_{\mathrm{b}}} \boldsymbol{a} \cdot\left(\boldsymbol{\Sigma}^{0} \mathbf{e}_{z}\right) \mathrm{d} \hat{\mathbf{x}}^{\prime}=\frac{\partial \mathcal{T}^{0}}{\partial \tilde{z}}=\mu_{\mathrm{b}} J_{\mathrm{b}} \frac{\partial^{2} \Omega^{1}}{\partial \tilde{z}^{2}} .
$$

Thus, $\Omega^{1}$ is independent of $\tilde{z}$ (a linear dependance w.r.t. $\tilde{z}$ is prevented by its $\tilde{z}$-periodicity), and $\mathcal{T}^{0}$ reduces to

$$
\mathcal{T}^{0}(\mathbf{x})=\mu_{\mathrm{b}} J_{\mathrm{b}} \frac{\partial \Omega^{0}}{\partial z}(\mathbf{x}), \quad \frac{\partial \Omega^{1}}{\partial \tilde{z}}=0
$$

Appendix A.4. Proof of $P_{3}: \Omega^{0}=0$ in 16

Here, we shall see that the equation for torsion (A.10) can be complemented to get the wave equation for $\Omega^{0}$; in addition that the associated boundary conditions impose $\Omega^{0}=0$. (From A.3 we already know that $\Omega^{0}(\mathbf{x})$ does not depend of $\tilde{z}$.)

Appendix A.4.1. The wave equation for torsion

We start with the derivation of the equation satisfied by the macroscopic rotation $\Omega^{0}$. We use the relation $\operatorname{div}_{\hat{\mathbf{x}}^{\prime}} \Sigma^{2}+\operatorname{div}_{\tilde{z}} \Sigma^{1}+\operatorname{div}_{\mathbf{x}} \Sigma^{0}+\rho_{\mathrm{b}} \omega^{2} \mathbf{V}^{0}=\mathbf{0}$ from $\left(\mathrm{E}_{a}\right)^{0}$ and $\left(\mathrm{E}_{z}\right)^{0}$ in $(13)$, that we multiply by $\boldsymbol{a}=\hat{y} \mathbf{e}_{x}-\hat{x} \mathbf{e}_{y}$ and integrate over $\left(\hat{\mathbf{x}}^{\prime}, \tilde{z}\right) \in \hat{\mathrm{s}}_{\mathrm{b}} \times\left(0, \tilde{h}_{\mathrm{F}}\right)$. We get

$$
\int_{\hat{\mathrm{s}}_{\mathrm{b}} \times\left(0, \tilde{h}_{\mathrm{F}}\right)} \boldsymbol{a} \cdot \operatorname{div}_{\mathbf{x}} \Sigma^{0} \mathrm{~d} \hat{\mathbf{x}}^{\prime} \mathrm{d} \tilde{z}+\rho_{\mathrm{b}} \omega^{2} \int_{\hat{\mathrm{s}}_{\mathrm{b}} \times\left(0, \tilde{h}_{\mathrm{F}}\right)} \boldsymbol{a} \cdot \mathbf{V}^{0} \mathrm{~d} \hat{\mathbf{x}}^{\prime} \mathrm{d} \tilde{z}=0,
$$

where we have accounted for $\int_{\hat{\mathrm{s}}_{\mathrm{b}}} \boldsymbol{a} \cdot \operatorname{div}_{\hat{\mathbf{x}}^{\prime}} \boldsymbol{\Sigma}^{2} \mathrm{~d} \hat{\mathbf{x}}^{\prime}=0$ as in A.9 and for the $\tilde{z}$-periodicity of $\boldsymbol{\Sigma}^{1}$. Next, owing to the form of $\mathbf{V}^{0}$ in A.2) (with $\Omega^{0}=\Omega^{0}(\mathbf{x})$ from A.3 ) and the form of $\Sigma^{0}$ in A.5 (with $\frac{\partial}{\partial z} \Omega^{1}=0$ from A.10), we obtain

$$
\frac{\partial \mathcal{T}^{0}}{\partial z}(\mathbf{x})+\rho_{\mathrm{b}} \omega^{2} J_{\mathrm{b}} \Omega^{0}(\mathbf{x})=0, \quad \rightarrow \quad \mu_{\mathrm{b}} \frac{\partial^{2} \Omega^{0}}{\partial z^{2}}(\mathbf{x})+\rho_{\mathrm{b}} \omega^{2} \Omega^{0}(\mathbf{x})=0,
$$

with $J_{\mathrm{b}}$ defined in A.8. 
At $z=h_{b}$. In the intermediate region located near $z=h_{\mathrm{b}}$, we introduce the torsion force $\left.\widetilde{\mathcal{T}}^{0}\right|_{h_{\mathrm{b}}}\left(\mathbf{x}^{\prime}, \tilde{z}\right)$. Repeating the same procedure than in Appendix A.3 in the region of the beam, we find that

$$
\left.\widetilde{\mathcal{T}}^{0}\right|_{h_{\mathrm{b}}}\left(\mathbf{x}^{\prime}\right)=\mu_{\mathrm{b}} J_{\mathrm{b}} \frac{\partial \widetilde{\Omega}^{0}}{\partial z}\left(\mathbf{x}^{\prime}\right), \quad \frac{\partial \widetilde{\Omega}^{1}}{\partial \tilde{z}}=0 .
$$

Next we go to the microscopic scale near $z=h_{\mathrm{b}}$ with the expansions (30). Multiplying $\left.\operatorname{div}_{\hat{\mathbf{x}}} \widehat{\boldsymbol{\Sigma}}^{0}\right|_{h_{\mathrm{b}}}=$ $\mathbf{0}$ by $\boldsymbol{a}=\hat{y} \mathbf{e}_{x}-\hat{x} \mathbf{e}_{y}$, integrating over $\widehat{\mathrm{X}}_{h_{\mathrm{b}}}=\left\{\hat{\mathbf{x}}^{\prime} \in \hat{\mathrm{s}}_{\mathrm{b}}, \hat{z} \in(-\infty, 0)\right\}$ and making use of the matching conditions and traction free boundary conditions, we deduce that

$$
\left.\widetilde{\mathcal{T}}^{0}\right|_{h_{\mathrm{b}}}\left(\mathbf{x}^{\prime}\right)=\lim _{\hat{z} \rightarrow-\infty} \int_{\hat{s}_{\mathrm{b}}}\left(\left.\hat{y} \widehat{\Sigma}_{x z}^{0}\right|_{h_{\mathrm{b}}}-\left.\hat{x} \widehat{\Sigma}_{y z}^{0}\right|_{h_{\mathrm{b}}}\right) \mathrm{d} \hat{\mathbf{x}}^{\prime}=0
$$

Finally, using the matching condition between the intermediate region and the inner region of the beams we get

$$
\mathcal{T}^{0}\left(\mathbf{x}^{\prime}, h_{\mathrm{b}}\right)=\left.\lim _{\tilde{z} \rightarrow-\infty} \widetilde{\mathcal{T}}^{0}\right|_{h_{\mathrm{b}}}\left(\mathbf{x}^{\prime}\right)=0
$$

At $z=0$. In the intermediate region located near $z=0$, in virtue of (14) which holds also for the intermediate expansions 24 , we have $\varepsilon^{\hat{\mathbf{x}}^{\prime}}\left(\left.\widetilde{\mathbf{V}}^{0}\right|_{0}\right)=\mathbf{0}$, hence $\left.\widetilde{V}_{x}^{0}\right|_{0}=\left.U_{x}^{0}\right|_{0}\left(\mathbf{x}^{\prime}, \tilde{z}\right)+\left.\widetilde{\Omega}^{0}\right|_{0}\left(\mathbf{x}^{\prime}\right) \hat{y}$. The goal is to show that $\left.\widetilde{\Omega}^{0}\right|_{0}\left(\mathbf{x}^{\prime}\right)=0$. For that, we go at the microscopic scale near $z=0$, where we recall (see section 3.2 .2 ) that since $\varepsilon^{\hat{\mathbf{x}}^{\prime}}\left(\left.\widehat{\mathbf{V}}^{0}\right|_{0}\right)=\mathbf{0},\left.\widehat{\mathbf{V}}^{0}\right|_{0}$ is a rigid body motion which can be reduced to a translation thanks to the periodic conditions acting at this scale. Therefore we get $\left.\widehat{V}_{x}^{0}\right|_{0}=\left.\widehat{U}_{x}^{0}\right|_{0}\left(\mathbf{x}^{\prime}\right)$. It now suffices to apply the micro-meso matching conditions i.e. $\left.\widetilde{V}_{x}^{0}\right|_{0}\left(\mathbf{x}^{\prime}, 0\right)=$ $\left.\lim _{\hat{z} \rightarrow+\infty} \widehat{V}_{x}^{0}\right|_{0}$, to show that that $\left.\widetilde{\Omega}^{0}\right|_{0}\left(\mathbf{x}^{\prime}\right)=0$. Finally, using again matching conditions between the intermediate region and the inner region of the beams we deduce that

$$
\Omega^{0}\left(\mathbf{x}^{\prime}, 0\right)=\left.\lim _{z \rightarrow+\infty} \widetilde{\Omega}^{0}\right|_{0}\left(\mathbf{x}^{\prime}\right)=0 .
$$

Given the wave equation (A.11) and the corresponding boundary conditions A.14 - A.15, we deduce that outside the set of torsional resonant frequencies which correspond to $\omega_{n}=\sqrt{\frac{\mu_{\mathrm{b}}}{\rho_{\mathrm{b}}}} \frac{n \pi}{2 h_{\mathrm{b}}}$ with $n \in \mathbb{Z}^{*}$, we have

$$
\Omega^{0}(\mathbf{x})=0
$$

224 Appendix A.5. Proof of $P_{4}: \frac{\partial U_{x}^{0}}{\partial z}=\frac{\partial U_{y}^{0}}{\partial z}=0$ in 16

Appendix A.5.1. Determination of $\mathbf{V}^{3}$ and $\mathbf{\Sigma}^{1}$

The first step is to solve the problem on $\left(\Sigma^{1}, V^{3}\right)$ which reads, from $\left(\mathrm{E}_{a}\right)^{-1}$ and $\left(\mathrm{E}_{z}\right)^{-1}$ in $(13)$ and from $\left(C^{\prime}\right)^{1}$ in (14),

$$
\left\{\begin{array}{c}
\operatorname{div}_{\hat{\mathbf{x}}^{\prime}} \boldsymbol{\Sigma}^{1}+\operatorname{div}_{\tilde{z}} \Sigma^{0}=\mathbf{0} \quad \text { in } \hat{\mathbf{S}}_{\mathrm{b}}, \quad \boldsymbol{\Sigma}^{1} \mathbf{n}=\mathbf{0} \quad \text { on } \partial \hat{\mathbf{s}}_{\mathrm{b}}, \\
\varepsilon^{\hat{\mathbf{x}}^{\prime}}\left(\mathbf{V}^{3}\right)+\varepsilon^{\tilde{z}}\left(\mathbf{V}^{2}\right)+\varepsilon^{\mathbf{x}}\left(\mathbf{V}^{1}\right)=\frac{1+v_{\mathrm{b}}}{E_{\mathrm{b}}} \boldsymbol{\Sigma}^{1}-\frac{v_{\mathrm{b}}}{E_{\mathrm{b}}} \operatorname{tr}\left(\boldsymbol{\Sigma}^{1}\right) I .
\end{array}\right.
$$


We short-circuit the proof by assuming, or anticipating, that

$$
\Sigma_{x x}^{1}=\Sigma_{x y}^{1}=\Sigma_{y y}^{1}=0
$$

which can be verified a posteriori. As a result, from A.17) and with $\operatorname{tr}\left(\Sigma^{1}\right)=\Sigma_{z z}^{1}$, we have $\Sigma_{z z}^{1}=E_{\mathrm{b}}\left(\frac{\partial V_{z}^{2}}{\partial \tilde{z}}+\frac{\partial V_{z}^{1}}{\partial z}\right)$. Next, using $V_{z}^{2}$ in A.4,$V_{z}^{1}$ in $(\mathrm{A} .3)$ and with $V_{z}^{0}=U_{z}^{0}(\mathbf{x})$ independent of $\tilde{z}$ from (A.2), we obtain

$$
\Sigma_{z z}^{1}=E_{\mathrm{b}}\left(\frac{\partial U_{z}^{2}}{\partial \tilde{z}}+\frac{\partial U_{z}^{1}}{\partial z}-\frac{\partial^{2}}{\partial \tilde{z}^{2}}\left(U_{x}^{1} \hat{x}+U_{y}^{1} \hat{y}\right)-2 \frac{\partial^{2}}{\partial z \partial \tilde{z}}\left(U_{x}^{0} \hat{x}+U_{y}^{0} \hat{y}\right)\right) .
$$

Let us consider now in A.17) (second line) the strains associated to the 2D vanishing stress components $\left(\Sigma_{x x}^{1}, \Sigma_{x y}^{1}, \Sigma_{y y}^{1}\right)$. We obtain

$$
E_{\mathrm{b}}\left(\frac{\partial V_{x}^{3}}{\partial \hat{x}}+\frac{\partial V_{x}^{1}}{\partial x}\right)=-v_{\mathrm{b}} \Sigma_{z z}^{1}, \quad E_{\mathrm{b}}\left(\frac{\partial V_{y}^{3}}{\partial \hat{y}}+\frac{\partial V_{y}^{1}}{\partial y}\right)=-v_{\mathrm{b}} \Sigma_{z z}^{1}, \quad \frac{\partial V_{y}^{3}}{\partial \hat{x}}+\frac{\partial V_{x}^{3}}{\partial \hat{y}}+\frac{\partial V_{y}^{1}}{\partial x}+\frac{\partial V_{x}^{1}}{\partial y}=0 .
$$

We find that there exists $\left(U_{x}^{3}(\mathbf{x}, \tilde{z}), U_{y}^{3}(\mathbf{x}, \tilde{z}), \Omega^{3}(\mathbf{x}, \tilde{z})\right)$ such that the displacements $\left(V_{x}^{3}, V_{y}^{3}\right)$ read

$$
\left\{\begin{aligned}
V_{x}^{3}= & U_{x}^{3}(\mathbf{x}, \tilde{z})+\Omega^{3}(\mathbf{x}, \tilde{z}) \hat{y}-\frac{\partial \Omega^{1}}{\partial x} \hat{x} \hat{y}-\frac{\partial \Omega^{1}}{\partial y} \hat{y}^{2}-\frac{\partial U_{x}^{1}}{\partial x} \hat{x}-\left(\frac{\partial U_{y}^{1}}{\partial x}+\frac{\partial U_{x}^{1}}{\partial y}\right) \frac{\hat{y}}{2} \\
& -v_{\mathrm{b}}\left(\frac{\partial U_{z}^{2}}{\partial \tilde{z}}+\frac{\partial U_{z}^{1}}{\partial z}\right) \hat{x}+v_{\mathrm{b}} \frac{\partial}{\partial \tilde{z}}\left(\left(\frac{\partial U_{x}^{1}}{\partial \tilde{z}}+2 \frac{\partial U_{x}^{0}}{\partial z}\right) \frac{\hat{x}^{2}-\hat{y}^{2}}{2}+\left(\frac{\partial U_{y}^{1}}{\partial \tilde{z}}+2 \frac{\partial U_{y}^{0}}{\partial z}\right) \hat{x} \hat{y}\right), \\
V_{y}^{3}= & U_{y}^{3}(\mathbf{x}, \tilde{z})-\Omega^{3}(\mathbf{x}, \tilde{z}) \hat{x}+\frac{\partial \Omega^{1}}{\partial y} \hat{x} \hat{y}+\frac{\partial \Omega^{1}}{\partial x} \hat{x}^{2}-\frac{\partial U_{y}^{1}}{\partial y} \hat{y}-\left(\frac{\partial U_{y}^{1}}{\partial x}+\frac{\partial U_{x}^{1}}{\partial y}\right) \frac{\hat{x}}{2} \\
& -v_{\mathrm{b}}\left(\frac{\partial U_{z}^{2}}{\partial \tilde{z}}+\frac{\partial U_{z}^{1}}{\partial z}\right) \hat{y}+v_{\mathrm{b}} \frac{\partial}{\partial \tilde{z}}\left(\left(\frac{\partial U_{x}^{1}}{\partial \tilde{z}}+2 \frac{\partial U_{x}^{0}}{\partial z}\right) \hat{x} \hat{y}+\left(\frac{\partial U_{y}^{1}}{\partial \tilde{z}}+2 \frac{\partial U_{z}^{0}}{\partial z}\right) \frac{\hat{x}^{2}-\hat{y}^{2}}{2}\right) .
\end{aligned}\right.
$$

The remaining part of the solution, $\left(\Sigma_{x z}^{1}, \Sigma_{y z}^{1}\right)$ and $V_{z}^{3}$, is more demanding. From A.17 with $\Sigma_{z z}^{0}$ in (A.5) and using that $\frac{\partial}{\partial \tilde{z}} U_{z}^{0}=\frac{\partial}{\partial \tilde{z}} U_{z}^{1}=0$ from (A.2) and A.6, these fields satisfy

$$
\left\{\begin{array}{l}
\frac{\partial \Sigma_{x z}^{1}}{\partial \hat{x}}+\frac{\partial \Sigma_{y z}^{1}}{\partial \hat{y}}=-\frac{\partial \Sigma_{z z}^{0}}{\partial z}=E_{\mathrm{b}} \frac{\partial^{3}}{\partial \tilde{z}^{3}}\left(U_{x}^{0} \hat{x}+U_{y}^{0} \hat{y}\right) \quad \text { in } \hat{\mathrm{s}}_{\mathrm{b}}, \\
\Sigma_{x z}^{1}=\mu_{\mathrm{b}}\left(\frac{\partial V_{z}^{3}}{\partial \hat{x}}+\frac{\partial V_{x}^{2}}{\partial \tilde{z}}+\frac{\partial V_{z}^{1}}{\partial x}+\frac{\partial V_{x}^{1}}{\partial z}\right), \quad \Sigma_{y z}^{1}=\mu_{\mathrm{b}}\left(\frac{\partial V_{z}^{3}}{\partial \hat{y}}+\frac{\partial V_{y}^{2}}{\partial \tilde{z}}+\frac{\partial V_{z}^{1}}{\partial y}+\frac{\partial V_{y}^{1}}{\partial z}\right), \\
\Sigma_{x z}^{1} n_{x}+\Sigma_{y z}^{1} n_{y}=0 \quad \text { on } \partial \hat{\mathrm{s}}_{\mathrm{b}} .
\end{array}\right.
$$

(We have used the constitutive equation $(C)^{1}$ instead of $\left(C^{\prime}\right)^{1}$ in the second line.) Now, we rearrange the constitutive behavior using (i) $\left(V_{x}^{2}, V_{y}^{2}\right)$ in A.4 (with $\Omega^{0}=0$ from A.16), (ii) $\left(V_{x}^{1}, V_{y}^{1}, V_{z}^{1}\right)$ in A.2 -A.3 and (iii) $\frac{\partial}{\partial \tilde{z}} U_{z}^{0}=0$ from A.2 resulting in

$$
\frac{1}{\mu_{\mathrm{b}}} \underbrace{\left(\begin{array}{c}
\Sigma_{x z}^{1}-\mu_{\mathrm{b}} S \hat{y} \\
\Sigma_{y z}^{1}+\mu_{\mathrm{b}} S \hat{x}
\end{array}\right)}_{\Sigma^{\prime}}=\nabla_{\hat{\mathbf{x}}^{\prime}} \underbrace{\left(V_{z}^{3}+V_{z}^{\prime}\right)}_{V^{\prime}}+v_{\mathrm{b}} \frac{\partial^{3} U_{x}^{0}}{\partial \tilde{z}^{3}}\left(\begin{array}{c}
\frac{\hat{x}^{2}-\hat{y}^{2}}{2} \\
\hat{x} \hat{y}
\end{array}\right)+v_{\mathrm{b}} \frac{\partial^{3} U_{y}^{0}}{\partial \tilde{z}^{3}}\left(\begin{array}{c}
\hat{\hat{y}} \hat{y} \\
\frac{-\hat{x}^{2}+\hat{y}^{2}}{2}
\end{array}\right),
$$


where

$$
\left\{\begin{aligned}
V_{z}^{\prime}= & \left(\frac{\partial U_{x}^{2}}{\partial \tilde{z}}+\frac{\partial U_{z}^{1}}{\partial x}+\frac{\partial U_{x}^{1}}{\partial z}\right) \hat{x}+\left(\frac{\partial U_{y}^{2}}{\partial \tilde{z}}+\frac{\partial U_{z}^{1}}{\partial y}+\frac{\partial U_{y}^{1}}{\partial z}\right) \hat{y} \\
& -\frac{\partial}{\partial \tilde{z}}\left(\frac{\partial U_{x}^{0}}{\partial x} \hat{x}^{2}+\frac{\partial U_{y}^{0}}{\partial y} \hat{y}^{2}+\left(\frac{\partial U_{y}^{0}}{\partial x}+\frac{\partial U_{x}^{0}}{\partial y}\right) \hat{x} \hat{y}\right), \\
S= & \frac{\partial \Omega^{2}}{\partial \tilde{z}}+\frac{\partial \Omega^{1}}{\partial z}-\frac{1}{2} \frac{\partial}{\partial \tilde{z}}\left(\frac{\partial U_{y}^{0}}{\partial x}-\frac{\partial U_{x}^{0}}{\partial y}\right) .
\end{aligned}\right.
$$

Basically, the idea is to work on the auxiliary displacement variable $V^{\prime}$ which has absorbed $V_{z}^{\prime}$ associated to a gradient. Thus, it is now sufficient to solve the problem on $\left(\boldsymbol{\Sigma}^{\prime}, V^{\prime}\right)$ which satisfy A.21) along with $\operatorname{div}_{\hat{\mathbf{x}}^{\prime}} \boldsymbol{\Sigma}^{\prime}=E_{\mathrm{b}} \frac{\partial^{3}}{\partial \tilde{z}^{3}}\left(U_{x}^{0} \hat{x}+U_{y}^{0} \hat{y}\right)$ and $\boldsymbol{\Sigma}^{\prime} \boldsymbol{n}=0$; by linearity, we can set

$$
\left\{\begin{array}{c}
\boldsymbol{\Sigma}^{\prime}\left(\mathbf{x}, \tilde{z}, \hat{\mathbf{x}}^{\prime}\right)=\frac{\partial^{3}}{\partial \tilde{z}^{3}} U_{x}^{0}(\mathbf{x}, \tilde{z}) \sigma^{x}\left(\hat{\mathbf{x}}^{\prime}\right)+\frac{\partial^{3}}{\partial \tilde{z}^{3}} U_{y}^{0}(\mathbf{x}, \tilde{z}) \sigma^{y}\left(\hat{\mathbf{x}}^{\prime}\right), \\
V^{\prime}\left(\mathbf{x}, \tilde{z}, \hat{\mathbf{x}}^{\prime}\right)=\frac{\partial^{3}}{\partial \tilde{z}^{3}} U_{x}^{0}(\mathbf{x}, \tilde{z}) v^{x}\left(\hat{\mathbf{x}}^{\prime}\right)+\frac{\partial^{3}}{\partial \tilde{z}^{3}} U_{y}^{0}(\mathbf{x}, \tilde{z}) v^{y}\left(\hat{\mathbf{x}}^{\prime}\right),
\end{array}\right.
$$

and solve

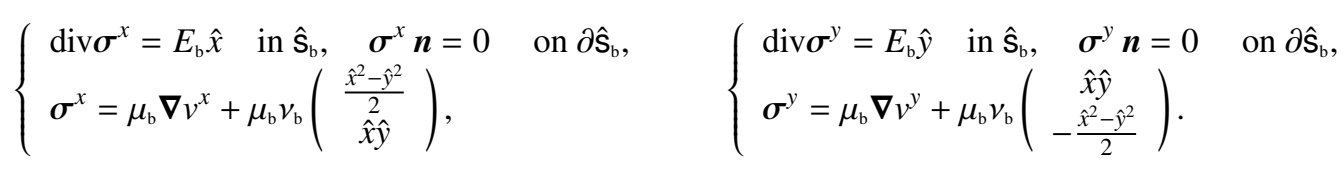

The above problems can be solved in polar coordinates with $\hat{x}=\hat{r} \cos \theta, \hat{y}=\hat{r} \sin \theta$ by looking for a solution of the form $v^{x}=g(\hat{r}) \cos \theta$ and $v^{y}=g(\hat{r}) \sin \theta$, with $g$ satisfying $g^{\prime \prime}+\frac{g^{\prime}}{\hat{r}}-\frac{g}{\hat{r}^{2}}=2 \hat{r}$, along with the boundary condition $\left(2 g^{\prime}\left(\hat{r}_{\mathrm{b}}\right)+v_{\mathrm{b}} \hat{r}_{\mathrm{b}}^{2}\right)=0$. It follows that

$$
g(\hat{r})=\frac{\hat{r}^{3}}{4}-\left(\frac{3}{4}+\frac{v_{\mathrm{b}}}{2}\right) \hat{r}_{\mathrm{b}}^{2} \hat{r}
$$

Eventually the solution reads

$$
\left\{\begin{array}{l}
V_{z}^{3}=-V_{z}^{\prime}+\frac{\partial^{3}}{\partial \tilde{z}^{3}}\left(\left(U_{x}^{0} \hat{x}+U_{y}^{0} \hat{y}\right) \frac{g}{\hat{r}}\right), \\
\frac{1}{\mu_{\mathrm{b}}} \Sigma_{x z}^{1}=S \hat{y}+\frac{\partial^{3}}{\partial \tilde{z}^{3}}\left[U_{x}^{0}\left(\left(\frac{g^{\prime}}{\hat{r}^{2}}+\frac{v_{\mathrm{b}}}{2}\right) \hat{x}^{2}+\left(\frac{g}{\hat{r}^{3}}-\frac{v_{\mathrm{b}}}{2}\right) \hat{y}^{2}\right)+U_{y}^{0}\left(\frac{g^{\prime}}{\hat{r}^{2}}-\frac{g}{\hat{r}^{3}}+v_{\mathrm{b}}\right) \hat{x} \hat{y}\right], \\
\frac{1}{\mu_{\mathrm{b}}} \Sigma_{y z}^{1}=-S \hat{x}+\frac{\partial^{3}}{\partial \tilde{z}^{3}}\left[U_{x}^{0}\left(\frac{g^{\prime}}{\hat{r}^{2}}-\frac{g}{\hat{r}^{3}}+v_{\mathrm{b}}\right) \hat{x} \hat{y}+U_{y}^{0}\left(\left(\frac{g^{\prime}}{\hat{r}^{2}}+\frac{v_{\mathrm{b}}}{2}\right) \hat{y}^{2}+\left(\frac{g}{\hat{r}^{3}}-\frac{v_{\mathrm{b}}}{2}\right) \hat{x}^{2}\right)\right],
\end{array}\right.
$$

226 with $\hat{r}=\sqrt{\hat{x}^{2}+\hat{y}^{2}}$ and with $\left(V_{z}^{\prime}, S\right)$ defined in $(A .22)$.

227 Appendix A.5.2. Macroscopic equation for the flexural motions $U_{a}^{1}$

Now, we want to derive the relations equivalent to 20, 21) and 221). To do so, we iterate the procedure conducted in $\$ 3.1 .5$ First, we obtain $M_{a}^{1}, a=x, y$, by integrating $\Sigma_{z z}^{1}$ (whose form is in (A.19) over $\hat{\mathbf{x}}^{\prime} \in \hat{\mathrm{S}}_{\mathrm{b}}$ after multiplication by $\hat{x}$ then by $\hat{y}$. Next, the equilibrium is obtained starting with $\left(\mathrm{E}_{a}\right)^{1}$ in (13): $\frac{\partial}{\partial \hat{x}} \Sigma_{x a}^{3}+\frac{\partial}{\partial \hat{y}} \Sigma_{y a}^{3}+\frac{\partial}{\partial \bar{z}} \Sigma_{a z}^{2}+\frac{\partial}{\partial z} \Sigma_{a z}^{1}+\rho_{\mathrm{b}} \omega^{2} V_{a}^{1}=0, a=x, y$ (since 
$\Sigma_{x a}^{1}=\Sigma_{y a}^{1}=0$ from (A.18), that we integrate over $\hat{\mathbf{x}}^{\prime} \in \hat{\mathrm{s}}_{\mathrm{b}}$; then we use $\Sigma_{a z}^{1}$ in A.24 and $V_{a}^{1}$ in A.2. Eventually, the relations between bending moments and shear forces are written from $\left(\mathrm{E}_{z}\right)^{0}$ in (13): $\frac{\partial}{\partial \hat{x}} \Sigma_{x z}^{2}+\frac{\partial}{\partial \hat{y}} \Sigma_{y z}^{2}+\frac{\partial}{\partial z} \Sigma_{z z}^{1}+\frac{\partial}{\partial z} \Sigma_{z z}^{0}+\rho_{\mathrm{b}} \omega^{2} V_{z}^{0}=0$ (since $\Sigma_{x z}^{0}=\Sigma_{y z}^{0}=0$ from (17)), that we integrate over $\hat{\mathbf{x}}^{\prime} \in \hat{\mathrm{s}}_{\mathrm{b}}$ after multiplication by $\hat{a}=\hat{x}, \hat{y}$; then, we use $\Sigma_{z z}^{0}$ in (17) and $V_{z}^{0}$ in A.2. We obtain

$$
\left\{\begin{array}{l}
M_{a}^{1}=E_{\mathrm{b}} I_{\mathrm{b}} \frac{\partial^{2} U_{a}^{1}}{\partial \tilde{z}^{2}}+2 E_{\mathrm{b}} I_{\mathrm{b}} \frac{\partial^{2} U_{a}^{0}}{\partial z \partial \tilde{z}}, \quad \frac{\partial T_{a}^{2}}{\partial \tilde{z}}+\rho_{\mathrm{b}} \omega^{2} \varphi U_{a}^{1}+\mu_{\mathrm{b}} A_{\mathrm{b}} \frac{\partial^{4} U_{a}^{0}}{\partial z \partial \tilde{z}^{3}}=0, \\
T_{a}^{2}+\frac{\partial M_{a}^{1}}{\partial \tilde{z}}+E_{\mathrm{b}} I_{\mathrm{b}} \frac{\partial^{3} U_{a}^{0}}{\partial z \partial \tilde{z}^{2}}=0,
\end{array}\right.
$$

with $A_{\mathrm{b}}=-\frac{1}{2}\left(1+v_{\mathrm{b}}\right) \pi r_{\mathrm{b}}^{4}$. The above system conduces to

$$
\frac{\partial^{4} U_{a}^{1}}{\partial \tilde{z}^{4}}-\kappa^{4} U_{a}^{1}=B_{\mathrm{b}} \frac{\partial^{4} U_{a}^{0}}{\partial z \partial \tilde{z}^{3}}, \quad a=x, y,
$$

with $\kappa^{4}=\frac{\rho_{\mathrm{b}} \varphi}{E_{\mathrm{b}} l_{\mathrm{b}}} \omega^{2}$ and $B_{\mathrm{b}}=\frac{\mu_{\mathrm{b}} A_{\mathrm{b}}}{E_{\mathrm{b}} l_{\mathrm{b}}}-3=-4$. If $U_{a}^{0}$ depend on $z$ (and we want to show that it is not the case), then $U_{a}^{0}$ solution of $(23)$ reads $U_{a}^{0}(\mathbf{x}, \tilde{z})=A_{a}(\mathbf{x}) \sin \kappa \tilde{z}+B_{a}(\mathbf{x}) \cos \kappa \tilde{z}$. Integrating A.25 over $\tilde{z} \in(0,2 \pi / \kappa)$ after multiplication by $\cos \kappa \tilde{z}$ (resp. by $\sin \kappa \tilde{z})$ and integrating by part four times provides $\frac{\partial}{\partial z} A_{a}=0$ (resp. $\frac{\partial}{\partial z} B_{a}=0$ ), which allows us to conclude that

$$
\frac{\partial U_{x}^{0}}{\partial z}=\frac{\partial U_{y}^{0}}{\partial z}=0
$$

\section{Appendix B. The case of a two-dimensional array of plates}

We provide in this section the effective model for the alternative situation of a periodic array of identical plates supported by a soil substrate. We transpose the notations of the beams case, assuming that the plates have a height $h_{\mathrm{b}}$, a thickness $2 r_{\mathrm{b}}$ and that the periodicity of the array is $\ell$. We denote $\left(\lambda_{\mathrm{b}}, \mu_{\mathrm{b}}, \rho_{\mathrm{b}}\right)$ the material parameters of the plates and $\left(\lambda_{\mathrm{s}}, \mu_{\mathrm{s}}, \rho_{\mathrm{s}}\right)$ those of the substrate. The same scaling as for the beams case is used, see (9). For in-plane incident wave, the resulting displacement fields remain in-plane with

$$
U_{y}=0, \quad \frac{\partial U_{x}}{\partial y}=\frac{\partial U_{z}}{\partial y}=0, \quad \sigma_{x y}=\sigma_{y z}=0 .
$$

Following the same asymptotic procedure as for the beams, we derive an effective model for the array of plates. In the substrate, the displacement field is governed by the classical balance of linear momentum equation and constitutive equation of the substrate. The array of the plates is replaced by frequency dependent boundary conditions which encapsulate both the longitudinal and flexural resonant motions of the plates

$$
\left\{\begin{array}{l}
\operatorname{div} \boldsymbol{\sigma}+\rho_{\mathrm{s}} \omega^{2} \mathbf{u}=\mathbf{0}, \quad \boldsymbol{\sigma}=2 \mu_{\mathrm{s}} \boldsymbol{\varepsilon}+\lambda_{\mathrm{s}} \operatorname{tr}(\boldsymbol{\varepsilon}) I, \quad \text { for } z \in(-\infty, 0), \\
\sigma_{x z}(x, 0)=\mu_{\mathrm{s}} k_{\mathrm{T}} f_{\mathrm{F}}\left(\omega, h_{\mathrm{b}}\right) u_{x}(x, 0), \quad \sigma_{z z}(x, 0)=\mu_{\mathrm{s}} k_{\mathrm{T}} f_{\mathrm{L}}\left(\omega, h_{\mathrm{b}}\right) u_{z}(x, 0),
\end{array}\right.
$$


and

$$
\begin{cases}f_{\mathrm{F}}\left(\omega, h_{\mathrm{b}}\right)=\varphi \frac{\rho_{\mathrm{b}}}{\rho_{\mathrm{s}}} \frac{k_{\mathrm{T}}}{\kappa} \frac{\operatorname{sh} \kappa h_{\mathrm{b}} \cos \kappa h_{\mathrm{b}}+\operatorname{ch} \kappa h_{\mathrm{b}} \sin \kappa h_{\mathrm{b}}}{1+\cos \kappa h_{\mathrm{b}} \operatorname{ch} \kappa h_{\mathrm{b}}}, & \kappa=\left(\frac{3 \rho_{\mathrm{b}} \omega^{2}}{E_{\mathrm{b}}^{*} r_{\mathrm{b}}^{2}}\right)^{1 / 4}, \\ f_{\mathrm{L}}\left(\omega, h_{\mathrm{b}}\right)=\varphi \frac{\rho_{\mathrm{b}}}{\rho_{\mathrm{s}}} \frac{k_{\mathrm{T}}}{K} \tan K h_{\mathrm{b}}, & K=\sqrt{\frac{\rho_{\mathrm{b}}}{E_{\mathrm{b}}^{*}}} \omega .\end{cases}
$$

with

$$
\varphi=\frac{2 r_{\mathrm{b}}}{\ell}, \quad E_{\mathrm{b}}^{*}=\frac{4 \mu_{\mathrm{b}}\left(\lambda_{\mathrm{b}}+\mu_{\mathrm{b}}\right)}{\lambda_{\mathrm{b}}+2 \mu_{\mathrm{b}}}=\frac{E_{\mathrm{b}}}{1-v_{\mathrm{b}}^{2}} .
$$

\section{References}

[1] J.-J. Marigo, K. Pham, A. Maurel, S. Guenneau, Effective model for elastic waves propagating in a substrate supporting a dense array of plates/beams with flexural resonances, Journal of the Mechanics and Physics of Solids.

[2] Y. Xiao, J. Wen, X. Wen, Flexural wave band gaps in locally resonant thin plates with periodically attached springmass resonators, Journal of Physics D: Applied Physics 45 (19) (2012) 195401.

[3] D. Torrent, D. Mayou, J. Sánchez-Dehesa, Elastic analog of graphene: Dirac cones and edge states for flexural waves in thin plates, Physical Review B 87 (11) (2013) 115143.

[4] A. Maznev, V. Gusev, Waveguiding by a locally resonant metasurface, physical Review B 92 (11) (2015) 115422.

[5] E. G. Williams, P. Roux, M. Rupin, W. Kuperman, Theory of multiresonant metamaterials for a 0 lamb waves, Physical Review B 91 (10) (2015) 104307.

[6] J. Y. Yoritomo, R. L. Weaver, P. Roux, M. Rupin, E. G. Williams, On band gap predictions for multiresonant metamaterials on plates, The Journal of the Acoustical Society of America 139 (3) (2016) 1282-1284.

[7] D. Colquitt, A. Colombi, R. Craster, P. Roux, S. Guenneau, Seismic metasurfaces: Sub-wavelength resonators and rayleigh wave interaction, Journal of the Mechanics and Physics of Solids 99 (2017) 379-393.

[8] N. Wootton, B. Erbas, J. Kaplunov, P. Wootton, Approximate analysis of surface wave-structure interaction, Journal of Mechanics of Materials and Structures 13 (2) (2018) 297-309.

[9] P. Wootton, J. Kaplunov, D. Colquitt, An asymptotic hyperbolic-elliptic model for flexural-seismic metasurfaces, Proceedings of the Royal Society A 475 (2227) (2019) 20190079.

[10] M. Lott, P. Roux, Effective impedance of a locally resonant metasurface, Physical Review Materials 3 (6) (2019) 065202.

[11] M. Lott, P. Roux, Locally resonant metamaterials for plate waves: the respective role of compressional versus flexural resonances of a dense forest of vertical rods, Fundamentals and Applications of Acoustic Metamaterials: From Seismic to Radio Frequency 1 (2019) 25-45.

[12] P. T. Wootton, J. Kaplunov, D. Prikazchikov, A second-order asymptotic model for rayleigh waves on a linearly elastic half plane, IMA Journal of Applied Mathematics 85 (1) (2020) 113-131.

[13] A. Colombi, P. Roux, S. Guenneau, P. Gueguen, R. V. Craster, Forests as a natural seismic metamaterial: Rayleigh wave bandgaps induced by local resonances, Scientific reports 6 (2016) 19238.

[14] M. Nieves, G. Carta, I. Jones, A. Movchan, N. Movchan, Vibrations and elastic waves in chiral multi-structures, Journal of the Mechanics and Physics of Solids 121 (2018) 387-408.

[15] A. Maurel, K. Pham, Multimodal method for the scattering by an array of plates connected to an elastic half-space, The Journal of the Acoustical Society of America, to appear. 